\title{
Sincronia Ventricular na Estimulação Cardíaca Parahissiana: Alternativa por Ativação Cardíaca Fisiológica (Estimulação Indireta do Feixe de His)?
}

\author{
Ventricular Synchrony in Para-Hisian Cardiac Pacing as an Alternative for Physiological Cardiac Activation \\ (Indirect Recruitment of the His Bundle?)
}

Andres Di Leoni Ferrari, ${ }^{10}$ Guilherme Ferreira Gazzoni, ${ }^{1}$ Luis Manuel Ley Domingues, ${ }^{1,2}{ }^{\circledR 0}$ Jessica Caroline Feltrin Willes, ${ }^{1}$ Gustavo Chiari Cabral, ${ }^{1}$ Flavio Vinicius Costa Ferreira, ${ }^{1}$ Laura Orlandini Lodi, ${ }_{1}^{1}$ Gustavo Reis ${ }^{3}$

Serviço de Cardiologia. Hospital São Lucas da Pontifícia Universidade Católica do Rio Grande do Sul (PUCRS), ${ }^{1}$ Porto Alegre, RS - Brasil Universidad Popular Autonoma del Estado de Puebla - Facultad de Medicina, ${ }^{2}$ Puebla - México

Eletrofisiologia Londrina, ${ }^{3}$ Londrina, PR - Brasil

\section{Resumo}

Fundamento: A estimulação cardíaca artificial (ECA) por captura direta ou indireta do feixe de His resulta em contração ventricular sincrônica (ECA fisiológica).

Objetivos: Comparar sincronia cardíaca, características técnicas e resultados de parâmetros eletrônicos entre duas técnicas de ECA indireta do feixe de His: a não seletiva e a parahissiana.

Métodos: Intervenção experimental (novembro de 2019 a abril de 2020) com implante de marca-passo definitivo (MPd) DDD em pacientes com fração de ejeção ventricular esquerda $>35 \%$. Foram comparadas a sincronia cardíaca resultante mediante algoritmo de análise eletrocardiográfica da variância espacial do QRS e as características técnicas associadas a cada método entre ECA hissiana não seletiva (DDD-His) e parahissiana (DDD-Var).

Resultados: De 51 pacientes (28 homens), 34 (66,7\%) foram alocados no grupo DDD-Var e 17 (33,3\%), no grupo DDDHis, com idade média de 74 e 79 anos, respectivamente. No grupo DDD-Var, a análise da variância espacial do QRS (índice de sincronia ventricular) mostrou melhora após o implante de MPd ( $p<0,001)$. Ao ECG pós-implante, 91,2\% dos pacientes do grupo DDD-Var mostraram padrão fisiológico de ECA, comprovando ativação similar à do DDD-His (88,2\%; $p=0,999$ ). O eixo do QRS estimulado também foi similar (fisiológico) para ambos os grupos. A mediana do tempo de fluoroscopia do implante foi de 7 minutos no grupo DDD-Var e de 21 minutos no DDD-His ( $<<0,001$ ), favorecendo a técnica parahissiana. A duração média do QRS aumentou nos pacientes do DDD-Var (114,7 ms pré-MPd e 128,2 ms pósimplante, $p=0,044)$. A detecção da onda $R$ foi de 11,2 $\mathrm{mV}$ no grupo DDD-Var e de 6,0 mV no DDD-His $(p=0,001)$.

Conclusão: A ECA parahissiana comprova recrutamento indireto do feixe de His, mostrando-se uma estratégia eficaz e comparável à ECA fisiológica ao resultar em contração ventricular sincrônica similar à obtida por captura hissiana não seletiva.

Palavras-chave: Marca-Passo Artificial; Estimulação Cardíaca Artificial; Terapia por Estimulação Elétrica.

Background:Artificial cardiac pacing by direct or indirect His bundle capture results in synchronous ventricular contraction (physiological pacing).

Objectives: To compare cardiac synchronization, technical characteristics, and electronic parameters between two techniques of indirect Hisbundle pacing: non-selective vs para-Hisian pacing.

Methods: The experimental intervention (between November 2019 and April 2020) consisted of implanting a DDD pacemaker in patients who had a left ventricular ejection fraction $>35 \%$. The resulting cardiac synchronization was compared using an electrocardiographic algorithm that analyzed QRS variation and the technical characteristics of non-selective Hisian pacing (DDD-His) and para-Hisian pacing (DDD-Var).

Results: Of 51 total patients (men: 28), 66.7\% (34) were allocated to the DDD-Var group and 33.3\% (17) to the DDD-His group. The mean ages in each group were 74 and 79 years, respectively. In the DDD-Var group, QRS variation (ventricular synchrony) improved after implantation $(p<0.001)$. In post-implantation ECG, $91.2 \%$ of the DDD-Var group presented a physiological pacing pattern, which was similar to the DDDHis group (88.2\%; $p=0.999$ ). The paced QRS axis was also similar (physiological) for both groups. Intraoperative fluoroscopy time during implantation was lower for the para-Hisian technique (median $7 \mathrm{~min}$ in the DDD-Var group vs 21 min in the DDD-His group, $p<0.001$ ). The

Correspondência: Andres Di Leoni Ferrari •

Hospital São Lucas da Pontificia Universidade Católica do Rio Grande do Sul - Avenida Ipiranga $66903^{\circ}$ Andar - Serviço de Cardiologia. CEP 90619-900, Porto Alegre, RS - Brasil

E-mail: dileoniferrari@gmail.com

Artigo recebido em 17/11/2020, revisado em 03/02/2021, aceito em 24/02/2021

DOI: https://doi.org/10.36660/abc.20201233 
mean QRS duration increased in the DDD-Var group (114.7 ms pre-implantation vs 128.2 ms post-implantation, $p=0.044$ ). The mean postimplantation R-wave amplitude was $11.2 \mathrm{mV}$ in the DDD-Var group vs $6.0 \mathrm{mV}$ in the DDD-His group, $p=0.001$.

Conclusion: Para-Hisian pacing appears to indirectly recruit the His bundle, which would make this an effective and comparable strategy for physiological pacing, resulting in synchronous ventricular contraction similar to that of non-selective Hisian pacing.

Keywords: Artificial Pacemaker; Artificial Cardiac Pacing; Electric Stimulation Therapy.

Full texts in English - http://www.arquivosonline.com.br

\section{Introdução}

A evolução da estimulação cardíaca artificial (ECA) demonstrou que a condução do impulso por meio da ativação muscular não fisiológica do ventrículo direito (VD), principalmente a estimulação apical (ECA "convencional"), está associada a efeitos cardíacos deletérios e repercussões negativas. ${ }^{1-4}$ A ECA convencional soluciona o problema elétrico e hemodinâmico ao restaurar a frequência cardíaca, porém causa alterações eletromecânicas decorrentes da dissincronia cardíaca. ${ }^{5}$ A dissincronia se manifesta eletricamente (QRS alargado com padrão de bloqueio de ramo esquerdo) e mecanicamente (remodelamento cardíaco, regurgitação mitral e disfunção sistólica). ${ }^{4,5}$

Vários estudos ratificaram a viabilidade e os resultados clínicos positivos da estimulação direta do feixe de His (direct His-bundle pacing, DHBP) quando comparada à ECA convencional. ${ }^{6-8}$ Atualmente, podemos considerar a indicação da DHBP para quase todos os distúrbios da condução cardíaca. A padronização dessa técnica, porém, é desafiadora. Alguns critérios ainda precisam ser refinados, tais como diferenças clínicas, se presentes, entre estimulação seletiva (selective His-bundle pacing, S-HBP) e não seletiva (nonselective His-bundle pacing, NS-HBP) ${ }^{9}$ do His; os maiores limiares de captura que resultam em desgaste acelerado da bateria do gerador; e os recursos adicionais (bainhas e eletrodos específicos) necessários para o posicionamento do eletrodo ventricular em contato com o feixe de His. ${ }^{10,11}$ Além disso, observa-se uma curva de aprendizado trabalhosa, com procedimentos mais longos, taxas de sucesso entre 60 e $90 \%$ e, em parte dos casos, dificuldades e complicações quanto à programação do dispositivo. ${ }^{10,12}$

A estimulação parahissiana (paraHisian pacing, PHP), por sua vez, apresenta uma curva de aprendizado mais rápida e de menor custo em termos de materiais, mostrando-se capaz de preservar, de modo similar, a sincronia da despolarização ventricular. ${ }^{12,13}$ A técnica consiste no posicionamento do eletrodo na região mais proximal do septo interventricular (IV) do VD, adjacente ao sistema de condução. Mais reprodutível, essa técnica é uma alternativa promissora para o conceito de estimulação cardíaca fisiológica ao indiretamente e rapidamente recrutar o sistema His-Purkinje, assemelhando-se à NS-HBP. ${ }^{12} \mathrm{O}$ objetivo deste estudo é analisar comparativamente a sincronia cardíaca obtida através de duas técnicas que envolvem uma abordagem indireta do sistema de condução para ECA fisiológica: a NS-HBP e a PHP.

\section{Metodologia}

Trata-se de um estudo de intervenção experimental realizado na Unidade de Estimulação Cardíaca e Ambulatório de MarcaPassos do Hospital São Lucas, da Pontifícia Universidade Católica do Rio Grande do Sul (PUCRS), em Porto Alegre, Brasil. Foram selecionados pacientes submetidos a implante de marca-passo definitivo (MPd) de dupla câmara DDD conforme as diretrizes vigentes, ${ }^{14}$ que possuíam fração de ejeção do ventrículo esquerdo (FEVE) preservada (> 50\%) ou intermediária (36 a 49\%). ${ }^{15}$ Todos os procedimentos de implante foram realizados pelo mesmo operador principal (ADLF). Todos os participantes assinaram o termo de consentimento livre e esclarecido. Excluíram-se pacientes com indicação de implante de cardiodesfibrilador, cardiodesfibrilador ou MPd multissítio ou MPd de câmara única e aqueles com dados incompletos para análise.

Os pacientes foram divididos em dois grupos: DDD-Var (implante de eletrodo VD parahissiano - PHP) e DDD-His (eletrodo VD em posição comprovada de captura do feixe de His não seletiva - NS-HBP), sendo o último guiado por mapeamento eletrofisiológico convencional.

A técnica de posicionamento do eletrodo do VD em posição proximal do septo IV para PHP seguiu metodologia previamente descrita. ${ }^{5,16-20}$ Brevemente, o eletrodo ventricular (cabos bipolares convencionais, de fixação ativa, de todos os fabricantes do mercado) foi montado em estilete guia manualmente moldado com curvatura ampla no terço distal, seguido de curvatura mais acentuada na porção final, com direcionamento posterior (Figura 1). 5,12

Guiado por anatomia radiológica, em projeção pósteroanterior, o eletrodo foi avançado para a artéria pulmonar e, com o fio guia totalmente inserido, foi tracionado para a via de saída do VD. Nessa projeção, o septo IV é dividido em três zonas: ${ }^{19}$ a superior (1/3 cranial do VD, entre o abaulamento da artéria pulmonar e o teto da valva tricúspide), a intermediária e a inferior (1/3 inferior do septo do VD). Em seguida, o posicionamento septal foi confirmado por incidência radioscópica em posição oblíqua anterior esquerda (entre 30 e 45 graus). Nessa incidência, o eletrodo está apontando perpendicularmente à coluna, em uma direção oposta à parede livre do VD ${ }^{12,19,20}$ (Figura 1).

Para confirmar a PHP (grupo DDD-Var), buscou-se o complexo QRS estimulado mais estreito (até $130 \mathrm{~ms}$ e sempre $<150 \mathrm{~ms}$ ) através do mapeamento do septo IV pelo eletrodo ventricular ${ }^{21}$ previamente à liberação do screw-in. Simultaneamente, em tempo real (intraoperatório), sob estimulação WVI decrementando desde amplitude $=5 \mathrm{~V}$ e largura de pulso $=1 \mathrm{~ms}$, o sistema de análise da variância espacial do QRS - dispositivo Synchromax ${ }^{\circledR}$ (Exo S.A. Argentina) - mostrava o índice imediato de sincronia (limeS). O sítio de PHP com o melhor índice era escolhido para a fixação definitiva do cabo do VD.

O limeS resulta do processamento gráfico e matemático do sinal promediado da variância cruzada das derivações DII (septo IV direito) e V6 (parede lateral do VE). Para essa análise, o dispositivo Synchromax ${ }^{\circledR}$ utiliza a medida do fluxo de corrente elétrica (volume e sentido) e a análise 

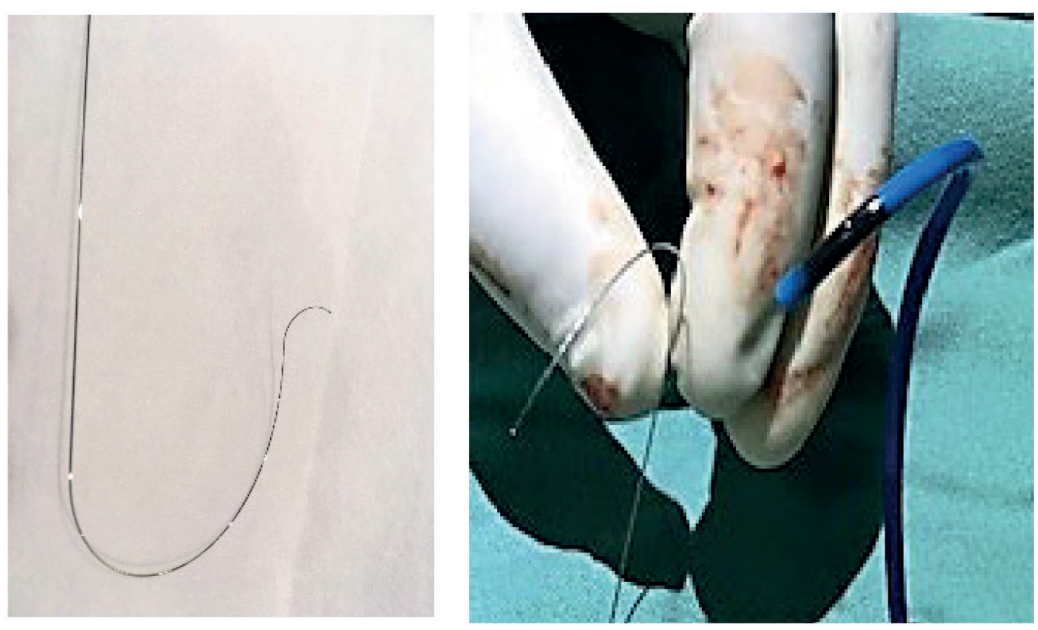

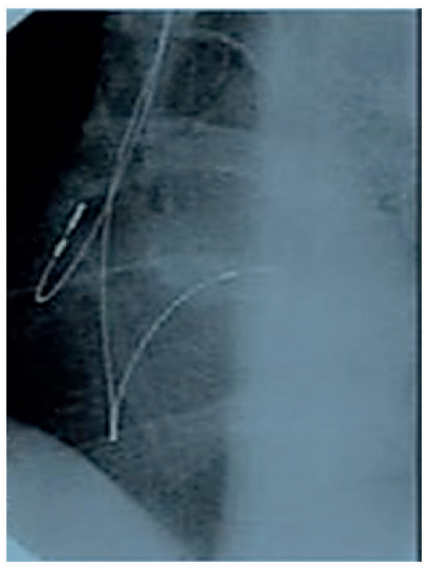

Silva Júnior e cols.

Sítios alternativos na estimulação cardíaca

Figura 1 - Esquerda: estilete guia manualmente moldado para direcionamento e implante de eletrodo do ventrículo direito (VD) no 1/3 proximal do septo interventricular para a estimulação parahissiana. Centro: Operador (ADLF) mostrando comparação do formato obtido pela moldagem da guia com a curvatura de uma das bainhas pré-moldada disponivel no Brasil (C315His Medtronic ${ }^{\mathrm{TM}}$ ). Direita: fluoroscopia obliqua esquerda mostrando a posição final do eletrodo do VD. Nota-se angulação da ponta, perpendicular à coluna. Adaptado de Ortega et al. e Júnior et al. 12,19

de concordância das deflexões intrinsecoides dos QRS (Figura 2A). ${ }^{12,22,23}$ Valores de limeS $<0,40$ são considerados normais (sincrônico); valores de limeS $>0,41$ e $<0,69$ são considerados dissincronia moderada; e valores de limeS $>0,7$ são considerados dissincronia severa (Figura 2B). 11,19,21

A abordagem e a captura do feixe de His (grupo DDDHis) foram feitas através de duas vias. Uma através da introdução femoral de cateter quadripolar para mapeamento eletrofisiológico e registro do potencial elétrico de His. A outra ocorreu através da introdução pela veia cefálica ou subclávia de bainhas defectíveis (C315-His, Medtronic, EUA) para o posicionamento do eletrodo de fixação ativa lumenless SelectSecure 3830 (Medtronic, EUA) em topografia de His, indicada pelo cateter de eletrofisiologia. Em seguida, confirmouse a captura do feixe de His seletiva (S-HBP) ou não seletiva (NS$\mathrm{HBP}){ }^{24}$ Simultaneamente e de forma não invasiva, durante o intraoperatorio, mediante estimulação VVI e decrementando a partir de energia de $5 \mathrm{~V}$ de amplitude $\mathrm{x} 1 \mathrm{~ms}$ de largura de pulso, realizou-se análise de variância espacial do QRS mediante dispositivo Synchromax ${ }^{\circledR}$ (Exo S.A. Argentina) determinando em tempo real o limeS, sob as mesmas características metodológicas descritas acima na estimulação PHP. Os melhores valores da NSHBP foram selecionados para estudo.

Além do limeS (Synchromax $\left.{ }^{\circledR}\right)$, foram registrados, para ambos os grupos, o tempo de fluoroscopia ( $R x)$ dedicado ao procedimento e eletrocardiograma (ECG) de superfície intraprocedimento e previamente à alta. A ativação endocárdica local do VD confirmou-se através dos seguintes parâmetros eletrônicos: medição da amplitude da onda $R$, teste de limiar de captura unipolar e bipolar por estimulação decremental em modo VVI e impedância unipolar e bipolar. Os melhores valores foram selecionados para análise.

A análise dos ECG era "cegada" ao tipo de metodologia e às características (pré e pós) do implante e do paciente. Para a determinação do eixo elétrico dos QRS do ECG pós-implante, considerou-se fisiológica a presença de ativação ventricular da direita para esquerda (QRS positivo nas derivações D1 e AVL) e de cima para baixo (QRS positivo em DII, DIII e AVF), assim como transição (onda $\mathrm{R} \geq \mathrm{S}$ ) até $\mathrm{V} 3-\mathrm{V} 4$ nas derivações precordiais. ${ }^{25}$ A presença de todos os três critérios categoriza um eixo fisiológico, a presença de dois critérios categoriza um eixo possivelmente fisiológico e a observação de somente um ou nenhum critério categoriza o eixo cardíaco como não fisiológico.

No grupo DDD-Var, para confirmar PHP sincrônica semelhante à NS-HBP e descartar estimulação muscular ventricular, utilizamos o modelo eletrocardiográfico proposto por Burri et al (Figura 3) verificando-se a combinação de ausência de plateau em D1, ausência de entalhe na derivação V1 e duração da ativação elétrica até o pico da onda R (RWPT) em V6 $<100$ ms. ${ }^{26-28}$ A presença dos três parâmetros indica ECA fisiológica semelhante à NS-HBP e descarta ativação ventricular puramente miocárdica. A presença de dois desses critérios indica ECA provavelmente fisiológica, enquanto a presença de apenas um critério indica ECA provavelmente não fisiológica. Quando todos os elementos estavam ausentes, considerou-se apenas captura do miocárdio (ECA miocárdica).

Para todos os pacientes, apenas na análise qualitativa, acompanhou-se a evolução clínica aguda (até a alta hospitalar) com interesse em complicações de causa cardiovascular, especialmente as relacionadas ao implante de MPd.

\section{Análise estatística}

As variáveis categóricas foram analisadas pelo teste exato de Fisher ou teste do qui-quadrado com correção de Yates, conforme distribuição das frequências nas diferentes categorias, e foram descritas por frequências e percentuais. A análise comparativa entre as variáveis categóricas, antes e depois do procedimento, foram associadas pelo teste de McNemar. As variáveis 


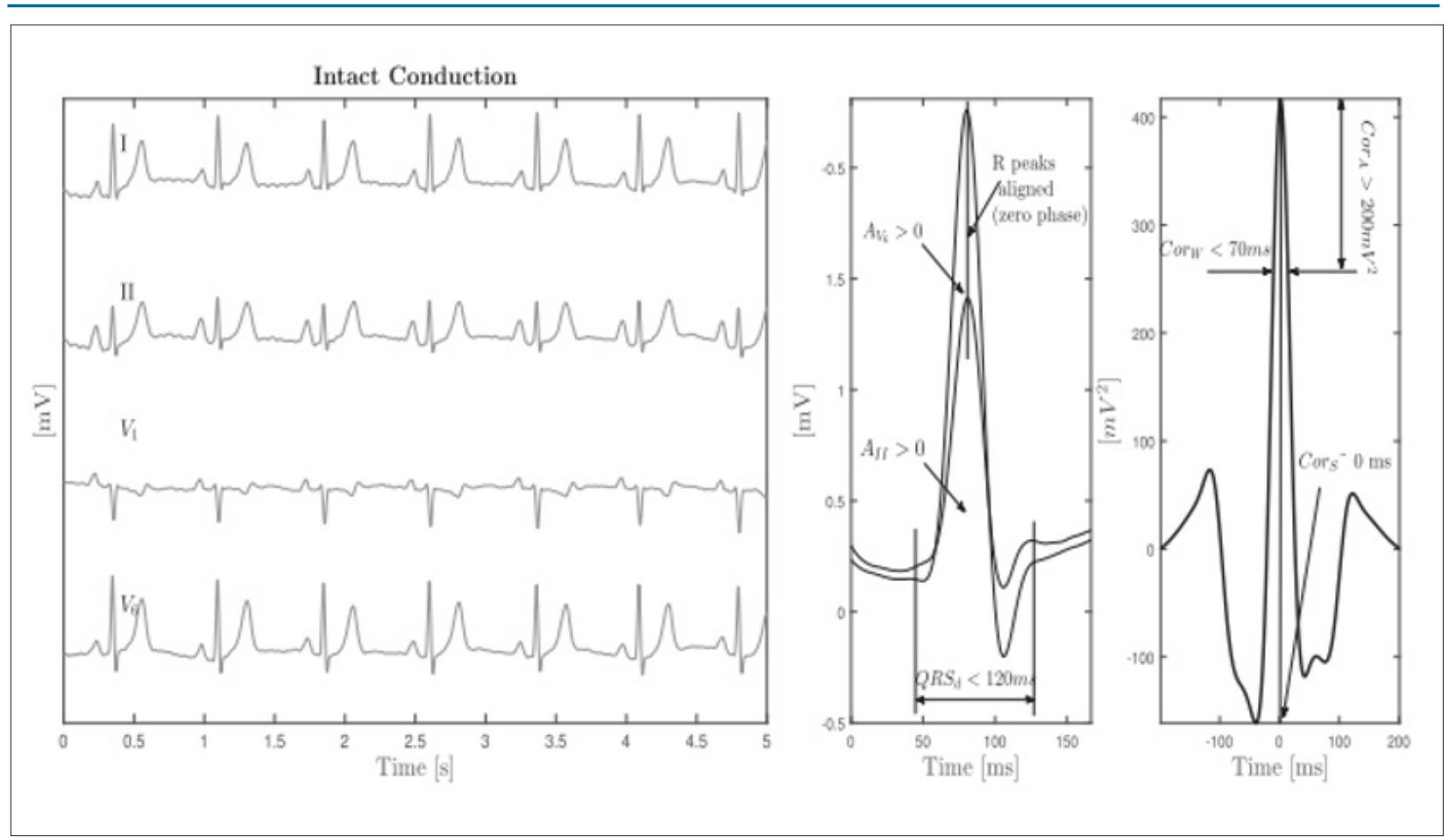

Figura $2 \mathrm{~A}$ - Correlação da variância espacial do QRS e valores normais para um paciente com condução intraventricular intacta (derivações I/ e $V_{6}$ ). Esquerda: traçados de eletrocardiograma. Centro: segmentos QRS sobrepostos (Ilars e $V_{6}^{\text {qrs }}$ ). Direita: análise de correlação cruzada das derivações II e $V_{6}$. Os picos dos complexos QRS coincidem, e o sinal de correlação cruzada mostra o seu máximo no tempo 0 (Cor $\left.{ }_{s}=0\right)$. Cor $_{s^{*}}$ deslocamento de correlação cruzada (ms); $\mathrm{Cor}_{W}$ : largura de correlação cruzada (ms); Cor $_{A^{\prime}}$ amplitude de correlação cruzada (mV); $A_{\| l}$ : área sob a derivação Il; $A V_{6}$ : área sob a derivação $V_{6}$ Adaptado de Bonomini et al..22

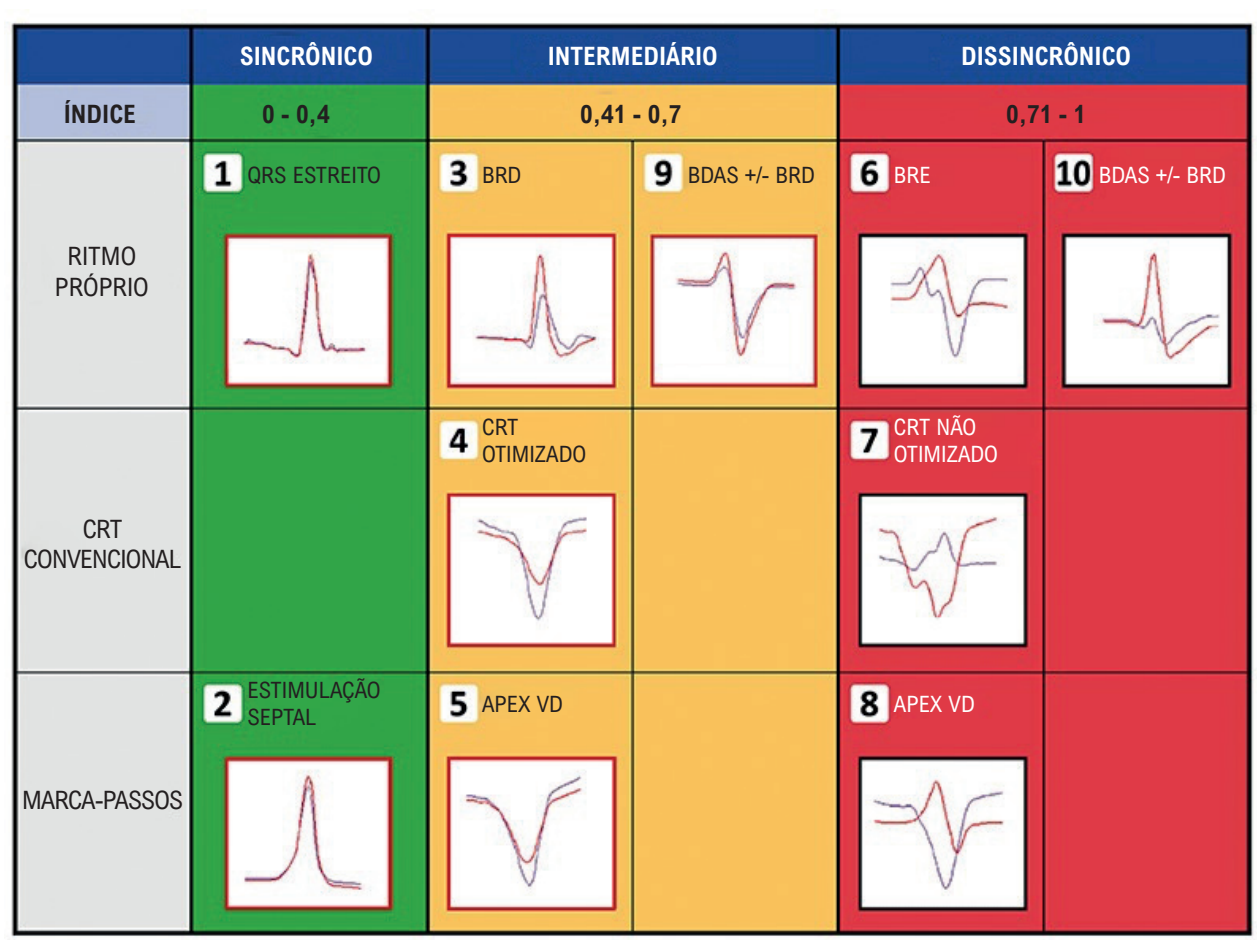

Figura 2B - Curvas com diferentes morfologias obtidas com o Synchromax ${ }_{T M}$ de acordo com o índice imediato de sincronia obtido em tempo real a partir do local de estimulação cardíaca artificial. Traços azuis: análise de variância do QRS da derivação Il. Traços vermelhos: análise da variância espacial do QRS da derivação $V_{6} B R D$ : Bloqueio de ramo direito; BRE: Bloqueio de ramo esquerdo; TRC: Terapia de Ressincronização Cardíaca; BDAS: Bloqueio Divisional ânterossuperior esquerdo; VD: ventrículo direito. 


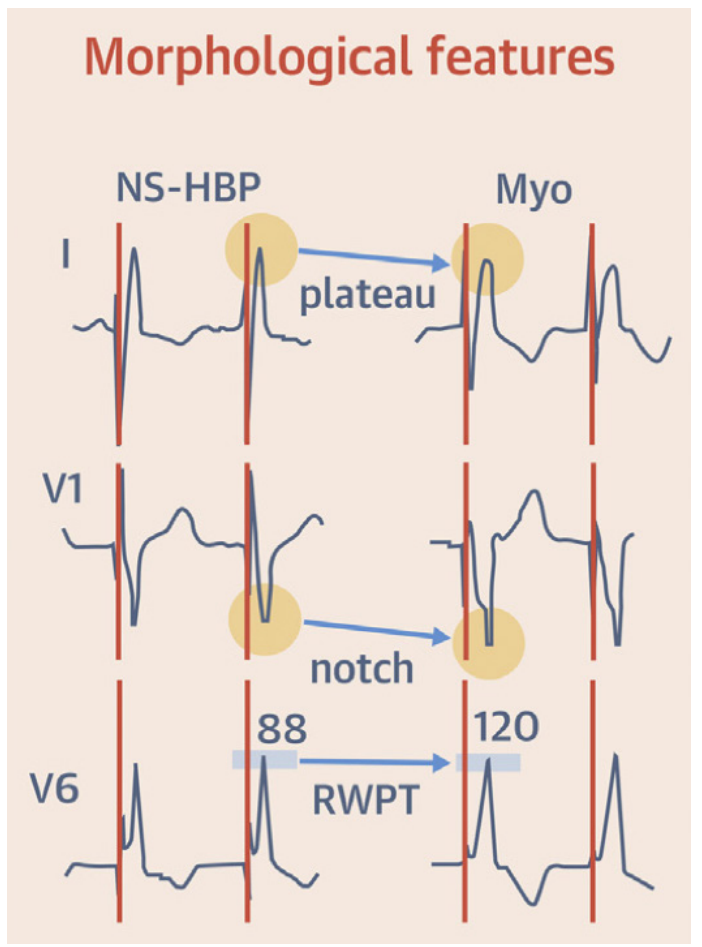

Figura 3 - Modelo eletrocardiográfico proposto por Burri et al. ${ }^{26}$ pela combinação de a) ausência de plateau em D1; b) ausência de entalhe (notch) na derivação V1; c) duração da ativação elétrica até o pico da onda R (RWPT) em V $6<100 \mathrm{~ms} \mathrm{~s}^{28-30}$ Os três parâmetros presentes $(a, b, c)$ indicam estimulação cardíaca artificial (ECA) fisiológica semelhante à NS-HBP e descartam ativação ventricular puramente miocárdica. A presença de dois critérios determina ECA provavelmente fisiológica, enquanto a presença de apenas um critério indica ECA provavelmente não fisiológica. $A$ ausência de todos os elementos denota captura puramente do miocárdio inespecífico (ECA miocárdica). ${ }^{28}$

quantitativas com distribuição simétrica foram comparadas pelo teste $t$ de Student para amostras independentes entre grupos; dentro dos grupos, foram comparadas pelo teste $t$ de Student para amostras emparelhadas. As variáveis com distribuição assimétrica foram comparadas pelo teste de Mann-Whitney entre os grupos e pelo teste de Wilcoxon dentro dos grupos. A simetria das variáveis quantitativas foi analisada pelo teste de Kolmogorov-Smirnov e descrita pela média e pelo desvio padrão; em caso de distribuição assimétrica, foi descrita pela mediana e pelos valores mínimo e máximo. Foi considerado um nível de significância de 5\% para as comparações estabelecidas. O software SPSS, versão 20.0, foi utilizado para a análise estatística.

\section{Resultados}

No período de novembro de 2019 a abril de 2020, foram incluídos 51 pacientes no estudo, dos quais 34 (66.7\%) foram alocados no grupo DDD-Var e 17 (33.3\%), no grupo DDD-His. A idade média foi de 74 anos para o primeiro grupo e de 79 anos para o segundo, sendo a maioria homens $(n=28)$. A etiologia mais prevalente para o implante de MPd foi o bloqueio atrioventricular total no grupo DDD-Var e a disfunção do nó sinusal (DNS) no grupo DDD-His. A FEVE era preservada (> 50\%) em 40 pacientes (78.4\%) e intermediária (36 a 49\%) em 11 (21.6\%) pacientes. A comparação dos grupos é apresentada na Tabela 1.

\section{Sincronia cardíaca}

$\mathrm{Na}$ amostra total, a análise da variância espacial do QRS (Synchromax $\left.{ }^{\circledR}\right)$ mostrou significância estatística $(p<0,001)$ do limeS na comparação do pré e do pós-operatório. Dos 20 pacientes sincrônicos no pré-operatório, 19 (95,0\%) permaneceram sincrônicos no pós-operatório. Dos 31 indivíduos restantes, cinco (9.8\%) eram intermediários (limeS = 0,41-0,69), e 26 (51\%) eram não sincrônicos (limeS > 0,7). Após o implante, 30 (96,8\%) tornaram-se sincrônicos, e apenas um (3.2\%) permaneceu intermediário.

Detalhadamente, dentro do grupo DDD-Var, também houve variação significativa na categoria de lime $S(p<0,001)$ entre o pré e o pós-implante. De 26 pacientes não sincrônicos, 25 (96,2\%) tornaram-se sincrônicos e apenas um (3,8\%), intermediário. Ainda, dos oito pacientes sincrônicos no pré-operatório, todos permaneceram sincrônicos pela medição do limeS no pós-operatório. No grupo DDD-His, dos 12 indivíduos sincrônicos, 11 (91,7\%) permaneceram sincrônicos, tendo apenas um paciente sido catalogado como não sincrônico no pós-operatório. Dos cinco pacientes restantes (não sincrônicos ou intermediários), todos (100\%) foram convertidos para sincrônicos no pós-operatório (Tabela 2).

A Tabela 2 também descreve as diferenças estatisticamente significativas entre os grupos no pré-operatório. O DDD-Var 
Di Leoni Ferrari et al. Estimulação Cardíaca Parahisiana é Fisiológica?

Artigo Original

\begin{tabular}{lccc}
\hline \multicolumn{1}{l}{ Tabela 1 - Comparação das características dos grupos } & $\begin{array}{c}\text { DDD-Var } \\
\mathbf{n}=\mathbf{3 4}\end{array}$ & $\begin{array}{c}\text { DDD-His } \\
\mathbf{n}=\mathbf{1 7}\end{array}$ & Valor de $\mathbf{p}$ \\
\hline Sexo masculino; $\mathrm{n}(\%)$ & $21(61,8)$ & $7(41,2)$ & 0,274 \\
\hline Idade em anos; média \pm DP & $74,0 \pm 8,9$ & $79,0 \pm 7,9$ & 0,063 \\
\hline Doença de base; $\boldsymbol{n}(\%)$ & & $\mathbf{0 , 0 0 4}$ \\
\hline BAVT & $17(50,0)^{\mathrm{a}}$ & $3(17,6)^{\mathrm{b}}$ & \\
\hline BAV 2 ${ }^{\circ}$ grau & $9(26,5)^{\mathrm{a}}$ & $3(17,6)^{\mathrm{a}}$ & \\
\hline DNS & $8(23,5)^{\mathrm{a}}$ & $11(64,7)^{\mathrm{b}}$ & \\
\hline $\begin{array}{l}\text { Fração de ejeção preservada } \\
(>50 \%) ; n(\%)\end{array}$ & $27(90,0)$ & $13(86,7)$ & 0,999 \\
\hline
\end{tabular}

BAV: bloqueio atrioventricular; BAVT: bloqueio atrioventricular total; DNS: disfunção do nó sinusal; DP: desvio padrão. Variáveis categóricas associadas pelo teste exato de Fisher ou teste do qui-quadrado com correção de Yates. Variáveis quantitativas associadas pelo teste $t$ de Student para amostras independentes. ${ }^{a, b}$ : letras diferentes indicam percentuais estatisticamente diferentes.

Tabela 2 - Comparação dos resultados pré e pós-implante do marca-passo definitivo

\begin{tabular}{|c|c|c|c|}
\hline & DDD-Var & DDD-His & Valor de $\mathrm{p}$ \\
\hline Índice de sincronia cardíaca & $n=34$ & $n=17$ & \\
\hline Pré-implante*; n (\%) & & & 0,001 \\
\hline Sincrônicos & $8(23,5)^{\mathrm{a}}$ & $12(70,6)^{b}$ & \\
\hline Intermediários & $3(8,8)^{\mathrm{a}}$ & $2(11,8)^{a}$ & \\
\hline Não sincrônicos & $23(67,6)^{\mathrm{a}}$ & $3(17,6)^{b}$ & \\
\hline Pós-implante; n (\%) & & & 0,560 \\
\hline Sincrônicos & $33(97,1)$ & $16(94,1)$ & \\
\hline Intermediários & $1(2,9)$ & - & \\
\hline Não sincrônicos & - & $1(5,9)$ & \\
\hline \multicolumn{4}{|l|}{ Valor do limeS } \\
\hline Pré-implante;; mediana (mínimo-máximo) & $1,00(0,12-1,00)$ & $0,21(0,06-1,00)$ & 0,001 \\
\hline Pós-implante; mediana (mínimo-máximo) & $0,18(0,11-0,70)$ & $0,18(0,11-0,72)$ & 0,461 \\
\hline \% variação; ; mediana (mínimo-máximo) & $-74(-89-192)$ & $0(-77-243)$ & $<0,001$ \\
\hline ECG pós-implante & $n=34$ & $n=17$ & \\
\hline Eixo; n (\%) & & & 0,074 \\
\hline Fisiológico & $26(76,5)$ & $8(47,1)$ & \\
\hline Possivelmente fisiológico & $8(23,5)$ & $9(52,9)$ & \\
\hline ECA & $n=34$ & $\mathrm{n}=17$ & \\
\hline Categoria (\%) & & & 0,915 \\
\hline Fisiológica & $15(44,1)$ & $6(35,3)$ & \\
\hline Provavelmente fisiológica & $16(47,1)$ & $9(52,9)$ & \\
\hline Provavelmente não fisiológica & $3(8,8)$ & $2(11,8)$ & \\
\hline Critério ausente de ECA fisiológica, $n$ (\%) & $n=19$ & $\mathrm{n}=11$ & \\
\hline RWPT $\geq 100 \mathrm{~ms}$ & $5(26,3)$ & $6(54,5)$ & 0,238 \\
\hline Plateau em D1 & $12(63,2)$ & $4(36,4)$ & 0,299 \\
\hline Notch em V1 & $5(27,8)$ & $3(27,3)$ & 0,999 \\
\hline
\end{tabular}

Sincrônicos: índice de sincronia cardíaca (limeS) $\leq 0,40$; intermediários: lime $S=0,41-0,70$; não sincrônicos: lime $S \geq 0,71$. Variáveis categóricas associadas pelo teste exato de Fisher ou teste do qui-quadrado com correção de Yates. Variáveis quantitativas com distribuição assimétrica associadas pelo teste de Mann-Whitney. †\% variação $=\left(\left[\right.\right.$ valor pós/pré valor pré]/valor pré) ${ }^{\star} 100$. a,b: letras diferentes indicam percentuais estatisticamente diferentes. ECA: estimulação cardíaca artificial; ECG: eletrocardiograma; RWPT: pico da onda R. tempo de ativação até pico da onda $R$ do QRS (ms). No "Critério ausente de ECA Fisiológica" um eventual paciente poderia mostrar mais de um critério. 
aglomerava mais pacientes não sincrônicos $(67,6 \%$ versus $17,6 \%$ no DDD-His) e menos pacientes sincrônicos (23,5\% versus 70,6\% no DDD-His). No pós-operatório, os grupos se assemelharam, uma vez que foi obtida sincronia em ambos os grupos (97,1\% no grupo DDD-Var e 94,1\% no DDD-His; $p=0,560$ ) (Figura 4). Os valores do limeS também foram significativamente diferentes entre os grupos no pré-operatório $(1,00$ no DDD-Var e 0,21 no DDD-His, $p=0,001)$ e similares no pós-operatório $(0,18$ no DDD-Var e 0,18 no DDD-His, $p=0,461$ ) (Figura 5), o que confirma a obtenção de ECA fisiológica com ambos os métodos, assemelhando a PHP à NS-HBP. O grupo DDD-Var teve redução mediana de $74 \%$ do limeS (versus redução mediana de $0 \%$ no grupo DDD-His, $p<0,001)$, sinalizando a magnitude da correção da sincronia. Analisando cada grupo separadamente e comparando os dados de sincronia entre o pré e o pós-operatório, o DDDVar demonstrou variação significativa (de 1,00 no pré para 0,18 no pós; $p<0,001$ ) e, conforme o esperado, não houve diferença significativa de variação no DDD-His (mediana de 0,21 no pré para 0,18 no pós-operatório; $p=0,453$ )

\section{Eixo elétrico fisiológico}

A Figura 6 demonstra que o eixo elétrico do QRS pós-implante é similar para ambos os grupos $(p=0,074)$. Corroborando a aproximação entre os métodos quanto ao recrutamento do sistema de condução de His-Purkinje, não houve diferença $(p=0,915)$ na comparação do eixo resultante como possivelmente fisiológico (47,1\% no DDD-Var e 52,9\% no DDD-His) ou fisiológico (44,1\% no DDD-Var e 35,3\% no DDD-His).
Estimulação cardíaca fisiológica: critérios de captura do sistema de condução

Como registrado na Tabela 2, ao analisarmos os critérios que sugerem captura comprovada do sistema de condução (descartando captura puramente miocárdica), 91,2\% dos pacientes do grupo DDD-Var apresentaram padrão fisiológico no pós-operatório (Figura 7), enquanto 88,2\% $(p=0,999)$ apresentaram no grupo DDD-His. O parâmetro RWPT $\geq 100 \mathrm{~ms}$ foi o critério que mais frequentemente descartou a possibilidade de ECA fisiológica no grupo DDD-His; no grupo DDD-Var, foi a presença de plateau em D1. A ECA foi catalogada como não fisiológica em três indivíduos do DDD-Var e em dois indivíduos do DDD-His.

\section{Duração do complexo QRS}

A Tabela 3 mostra que a duração média do QRS (ms) foi significativamente maior (Figura 8) no grupo DDD-Var do que no DDD-His tanto no pré $(114,7 \mathrm{~ms}$ versus $87,1 \mathrm{~ms}$, $\mathrm{p}=0,001)$ quanto no pós-implante (128,2 ms versus $102,1 \mathrm{~ms}$, $p<0,001)$. A mediana do QRS variou em $11 \%$ no grupo DDD-Var e em 20\% no grupo DDD-His ( $p=0,436)$. Quando comparada a média resultante após o implante, a duração do QRS aumentou significativamente em ambos os grupos $(114,7$ versus 128,2 ms no DDD-Var, $\mathrm{p}=0,044$; e 87,1 versus 102,1 ms no DDD-His, $p=0,003$ ).

\section{Tempo de uso de Rx e parâmetros eletrônicos pós-implante}

A mediana de tempo de Rx foi significativamente menor no grupo DDD-Var (7 minutos) do que no DDD-His (21 minutos) $(p<0,001)$, conforme a Figura 9. Os parâmetros

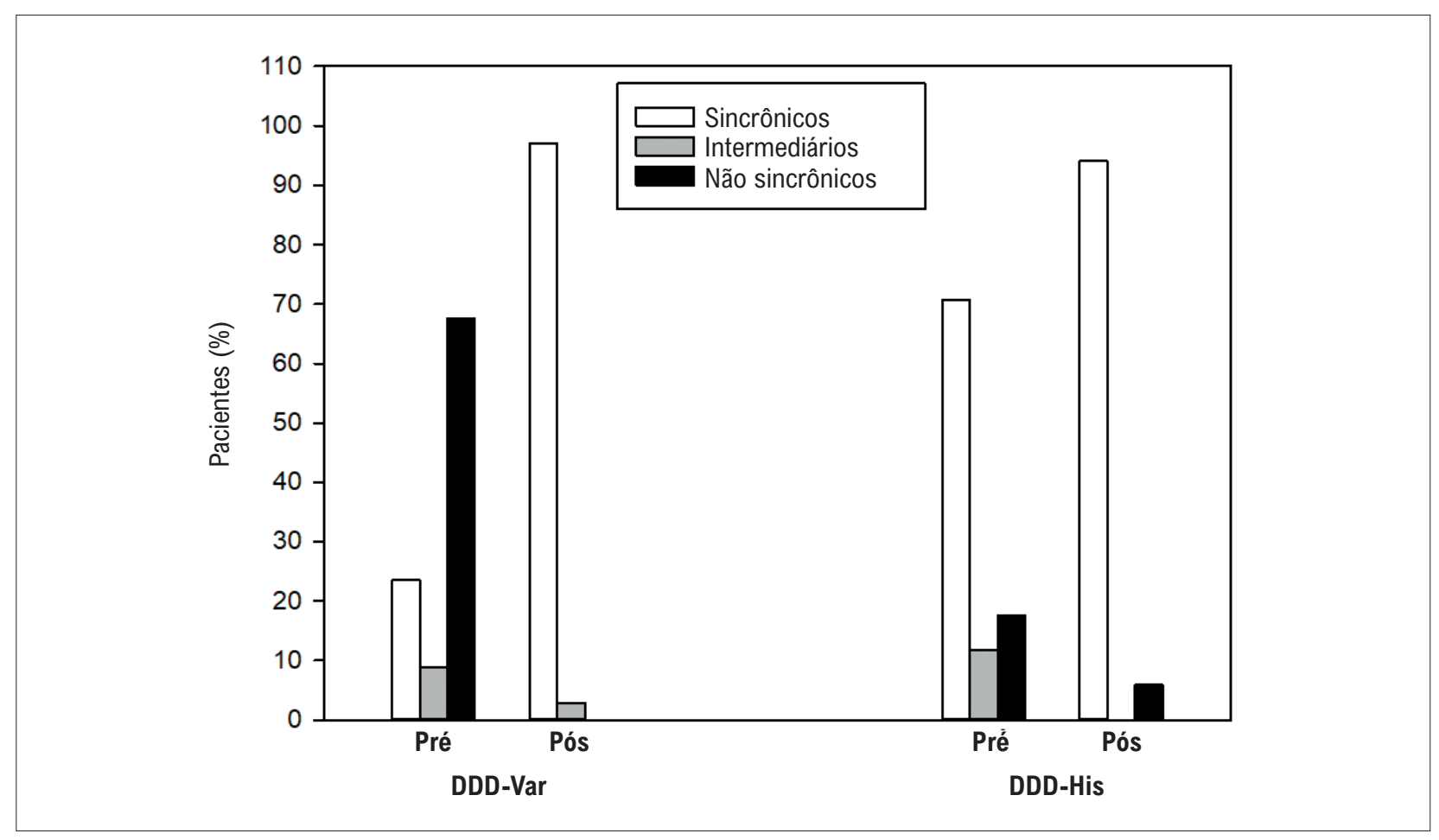

Figura 4 - Frequência de distribuição das categorias de sincronia cardíaca pré e pós-operatória entre os grupos. 


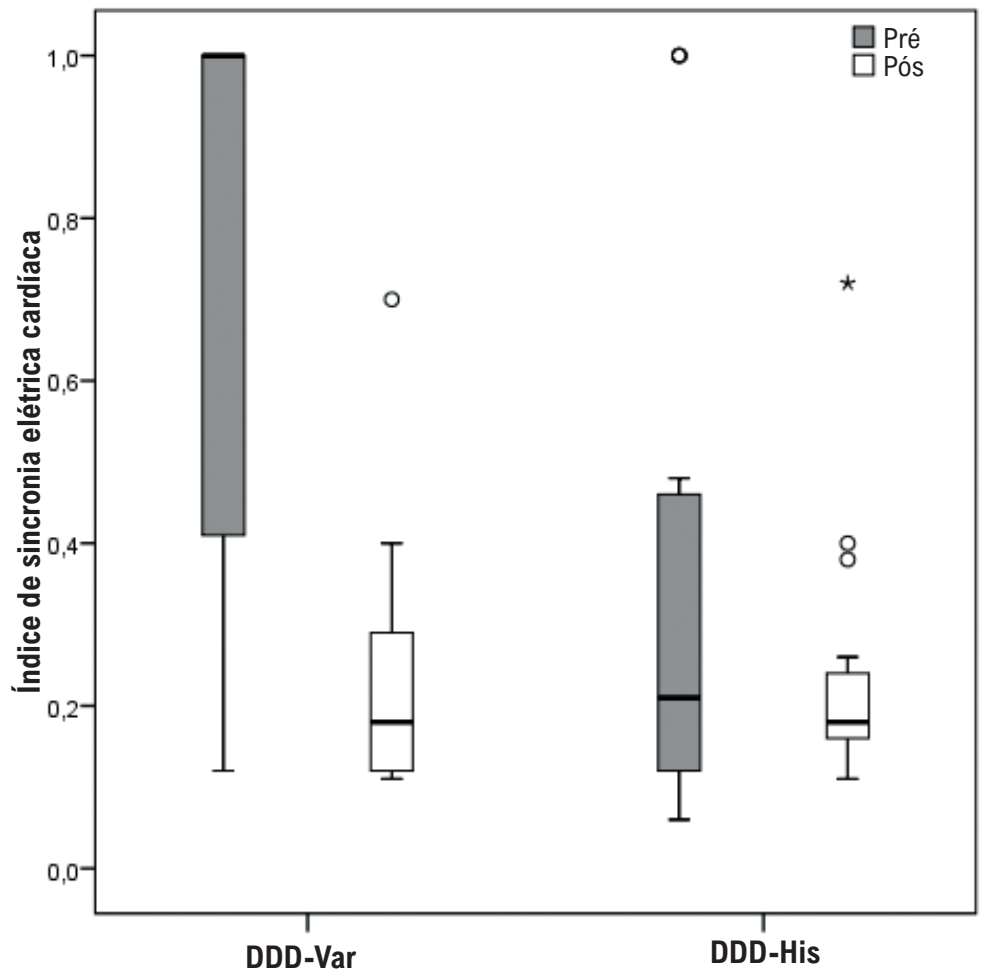

Figura 5 - Comparação do índice imediato de sincronia elétrica cardiaca entre os grupos.

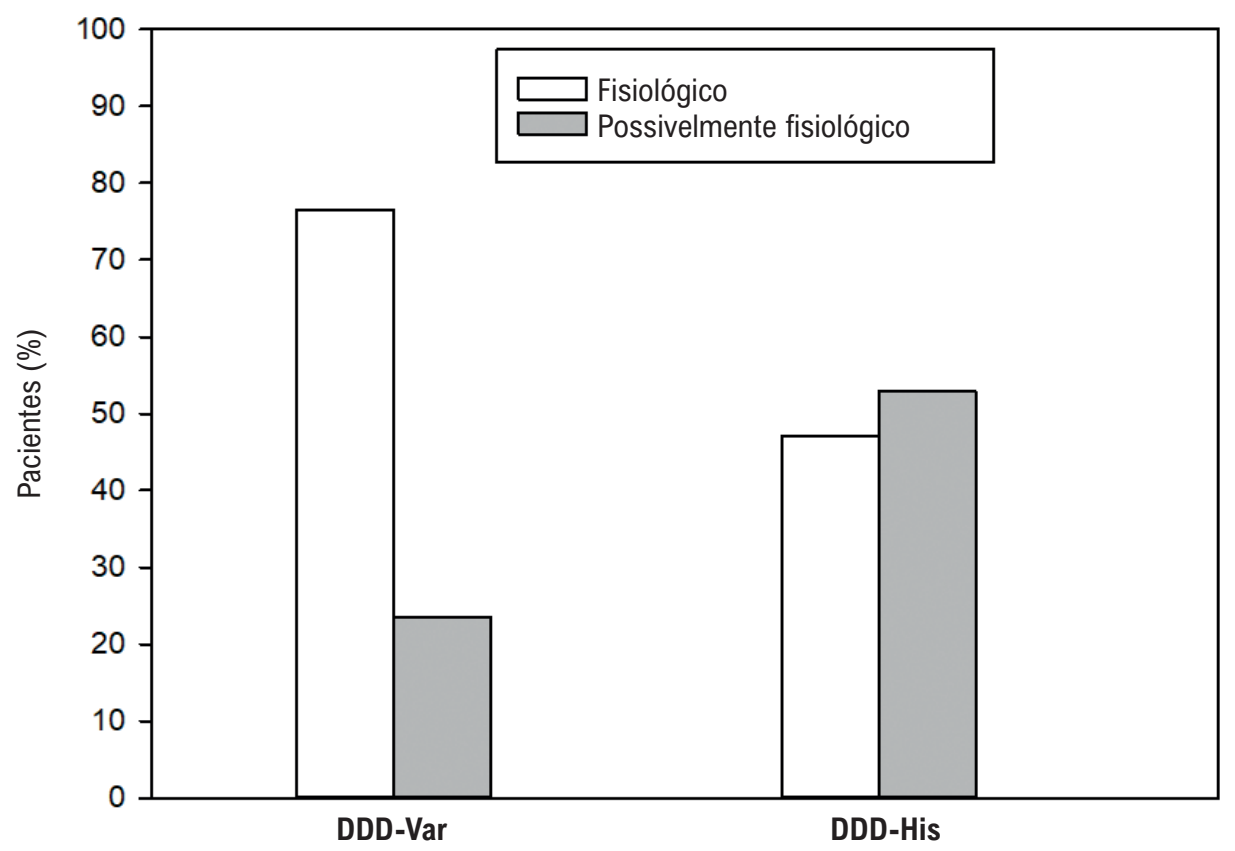

Figura 6 - Gráfico comparativo do eixo do eletrocardiograma entre os grupos. 


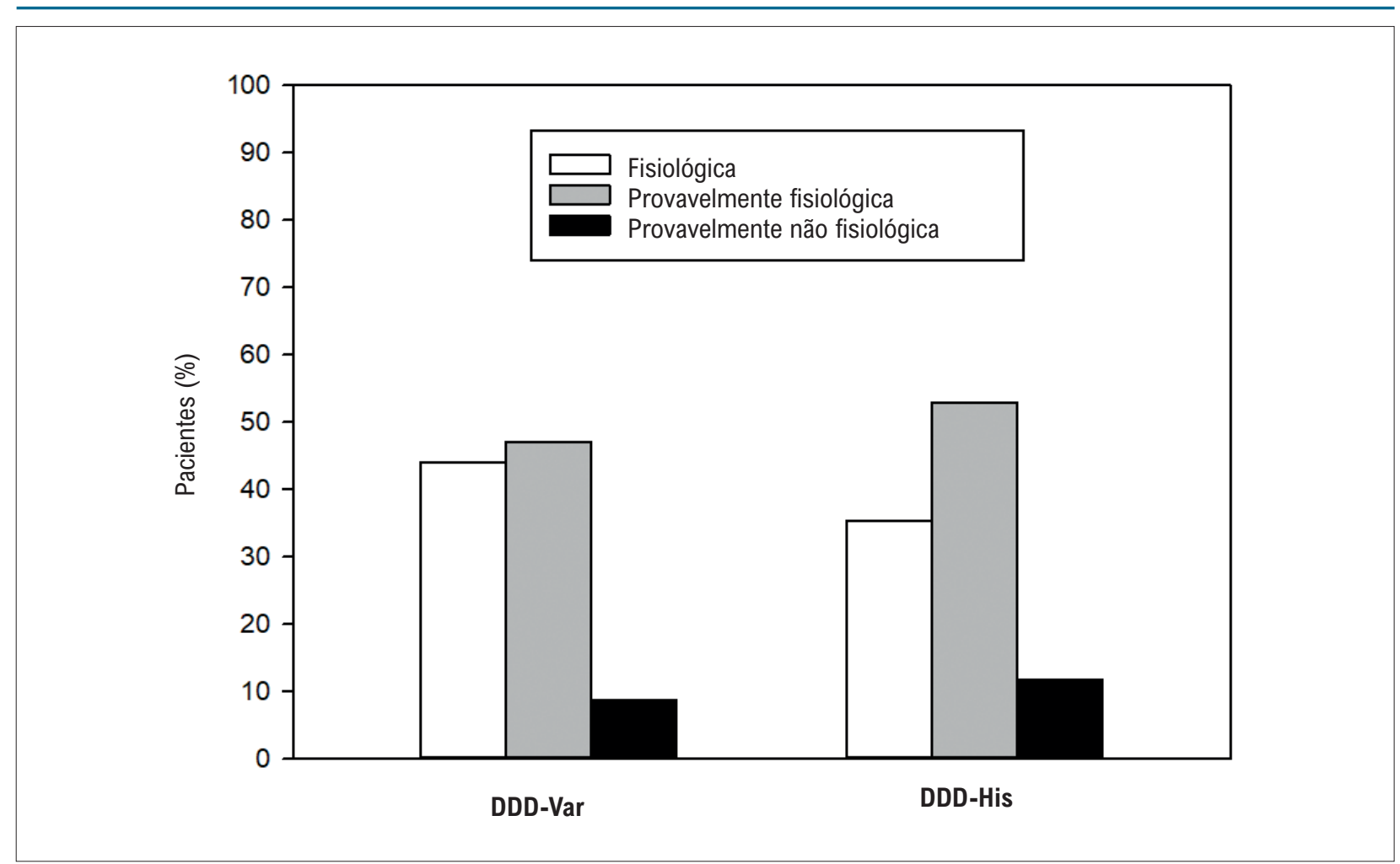

Figura 7 - Gráfico comparativo da sincronia cardiaca (limes) obtida com ambos métodos de estimulação cardíaca artificial

Tabela 3 - Comparação entre as técnicas indiretas de abordagem do feixe de His e as características pós-implante

\begin{tabular}{|c|c|c|c|}
\hline & DDD-Var & DDD-HIS & Valor de $p$ \\
\hline & $n=34$ & $n=17$ & \\
\hline \multicolumn{4}{|l|}{ QRS (ms) } \\
\hline Pré-implante; média \pm DP & $114,7 \pm 27,6$ & $87,1 \pm 21,6$ & 0,001 \\
\hline Pós-implante; média \pm DP & $128,2 \pm 16,2$ & $102,1 \pm 14,0$ & $<0,001$ \\
\hline \% variação; mediana (mínimo-máximo) & $11(-35$ a 138$)$ & $20(-14$ a 66$)$ & 0,436 \\
\hline Tempo de Rx; mediana (mínimo-máximo) & 7 (3 a 27$)$ & 21 (9 a 52) & $<0,001$ \\
\hline Limiar ventricular Uni/Bi; mediana (mínimo-máximo) & $0,6(0,4$ a 2,0$)$ & $0,9(0,3$ a 3,4$)$ & 0,074 \\
\hline Impedância ventricular Uni/Bi; média \pm DP & $754,8 \pm 262,2$ & $654,9 \pm 234,1$ & 0,190 \\
\hline Ondas R ventricular Uni/Bi; média $\pm \mathrm{DP}$ & $11,2 \pm 5,7$ & $6,0 \pm 3,8$ & 0,001 \\
\hline Complicações relacionadas ao implante do MPd & - & 1 & - \\
\hline
\end{tabular}

Variáveis categóricas associadas pelo teste exato de Fisher. Variáveis quantitativas com distribuição simétrica associadas pelo teste $t$ de Student para amostras independentes e assimétrica pelo teste de Mann-Whitney. DP: desvio padrão; MPd: marca-passo definitivo; Rx: fluoroscopia; Uni/Bipolar: estimulação unipolar ou bipolar - parâmetro mais favorável

de estimulação apresentaram medianas e distribuições aproximadas entre os grupos (Tabela 3). O limiar para o DDD-Var foi de 0,6 V, enquanto para o DDD-His foi de 0,9 $\mathrm{V}(\mathrm{p}=0,074)$. Os valores de impedâncias ventriculares foram de 754,8 ohms para o DDD-Var e de e 654,9 ohms para o DDD-His $(p=0,19)$. Entretanto, a média de amplitude das ondas R (Figura 10) favoreceu significativamente o grupo DDD-Var $(11,2 \mathrm{mV})$ em contraste ao DDD-His $(6,0 \mathrm{mV})$ $(p=0,001)$.
Acompanhamento pós-operatório (complicações agudas)

Apenas um paciente (grupo DDD-His) apresentou complicação relacionada ao MPd: pré-síncope por perda de captura por aumento de limiar do eletrodo VD.

\section{Discussão}

A captura e ativação do feixe de His é atualmente considerada o padrão-ouro da ECA fisiológica. ${ }^{8,14,24,29}$ A terminologia de 


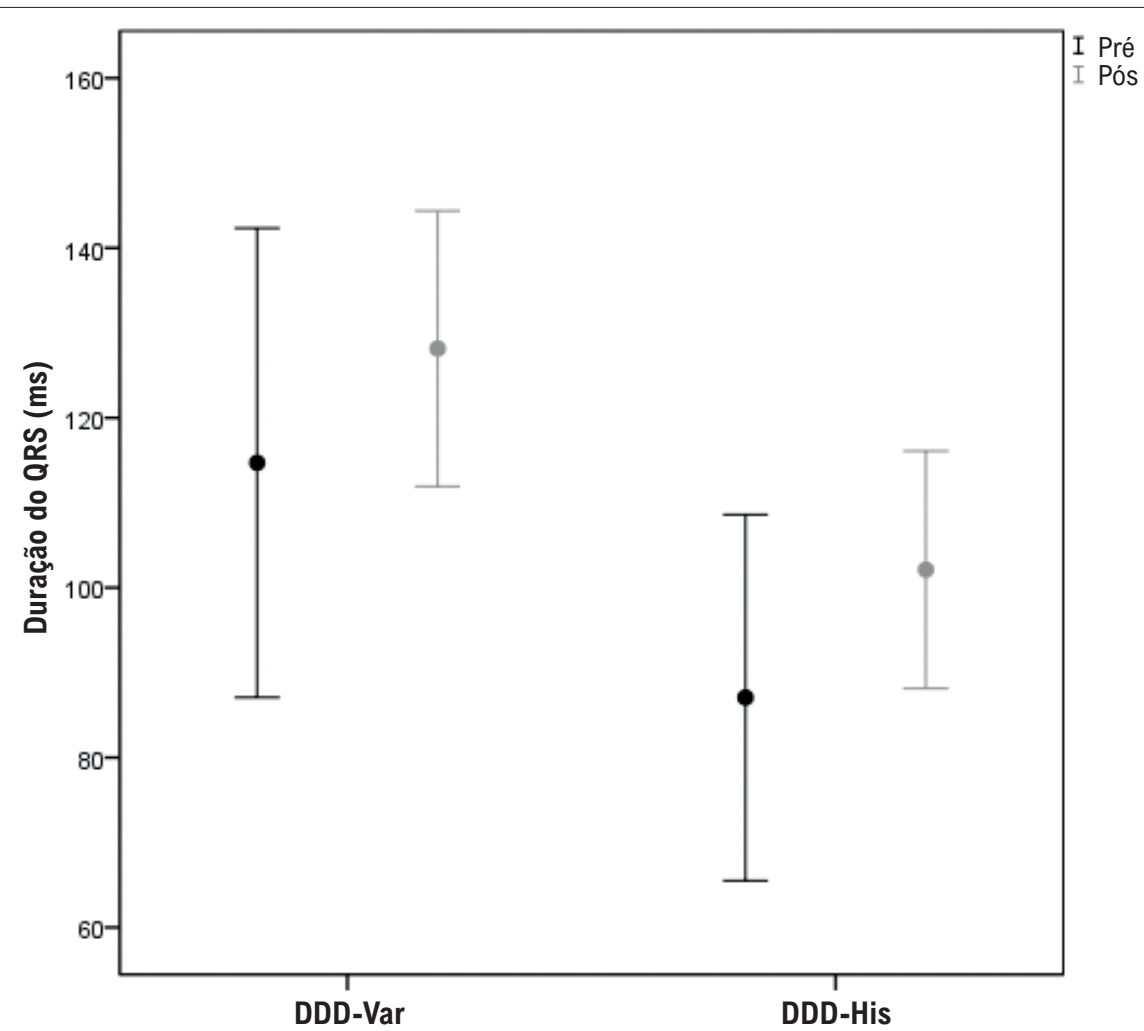

Figura 8 - Diferenças de duração do complexo QRS pré e pós-implante entre os grupos.

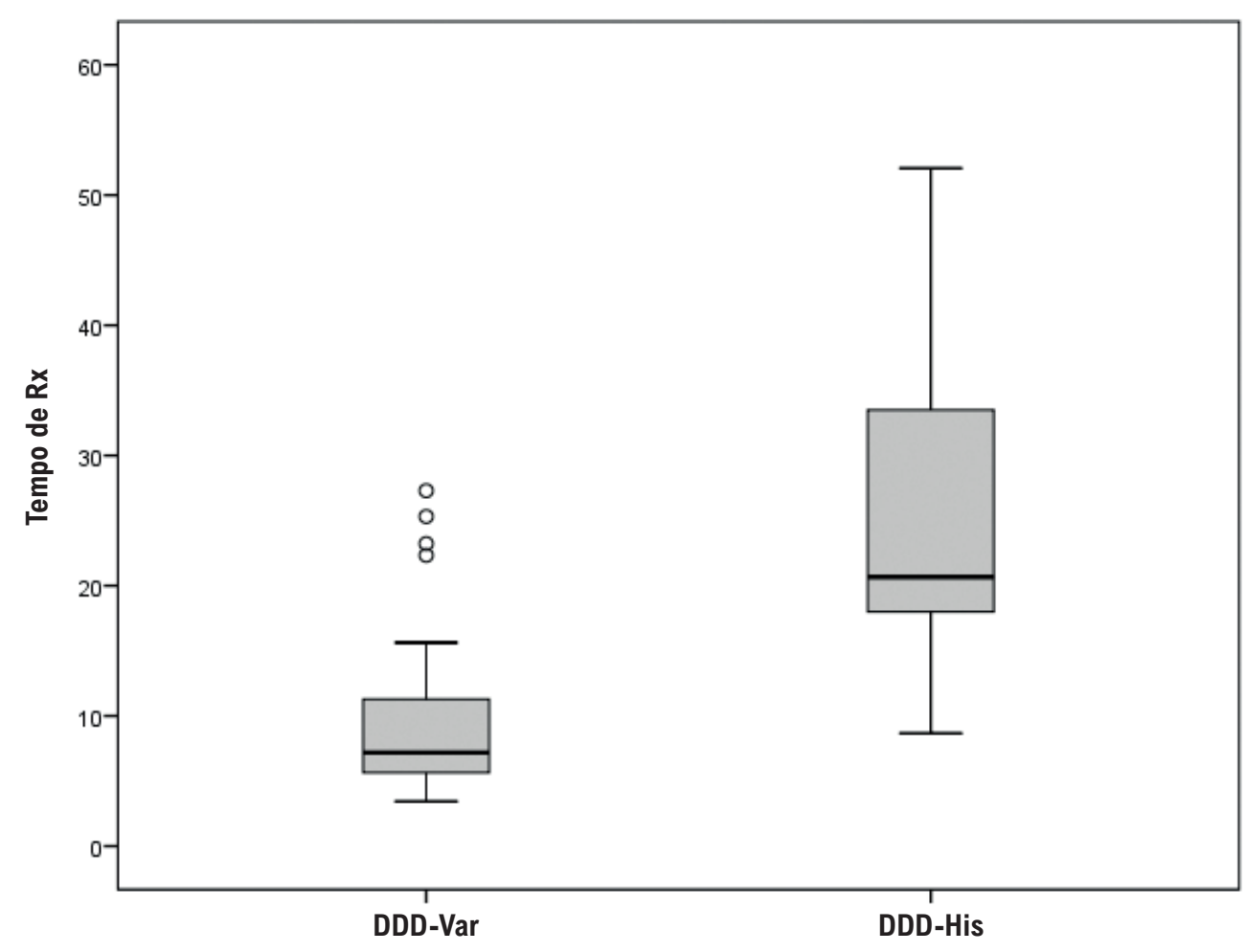

Figura 9 - Comparativo das médias de uso de fluoroscopia (Rx) entre os grupos. 


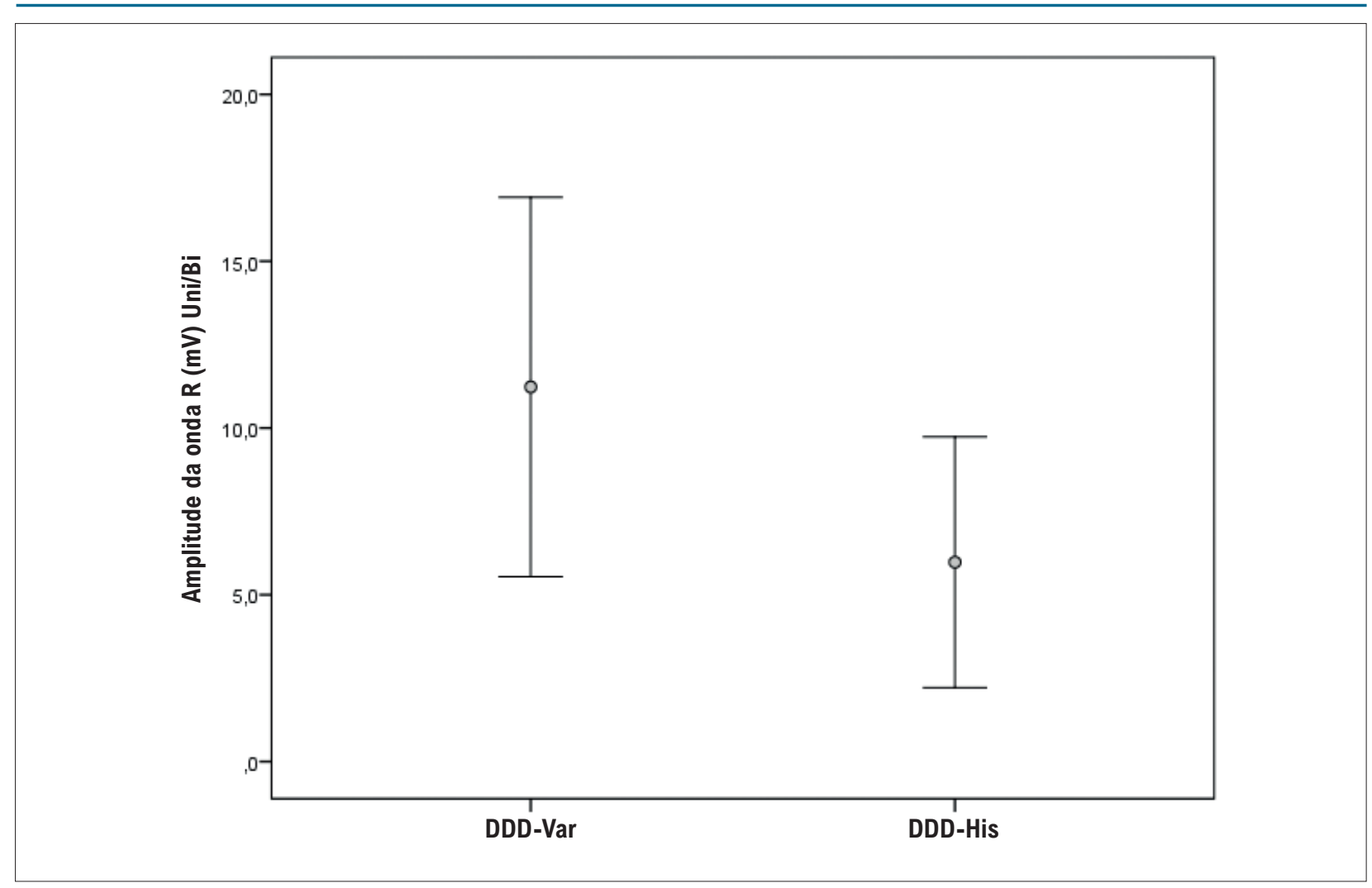

Figura 10 - Diferença da amplitude detectada nas ondas $R$ entre os dois grupos.

ECA parahissiana foi cunhada desde os primeiros resultados das tentativas de recrutar artificialmente o sistema eletrofisiológico intrínseco para reproduzir a contração cardíaca nativa. ${ }^{28}$ Este estudo mostra que a PHP se aproxima da captura não seletiva do feixe de His (NS-HBP) em termos de ativação homogênea ventricular (sincronia cardíaca) resultante da condução elétrica que determina a contração ventricular. Essa sincronia obtida pela técnica de PHP, confirmada por estritos critérios eletrocardiográficos e refinada pela análise da variância espacial do QRS (Synchromax®), torna a PHP uma alternativa viável, eficaz, reprodutível e de menor custo em comparação à NS-HBP.

\section{Sincronia cardíaca determinada pela variância espacial do QRS (limeS)}

A rápida e sincronizada transmissão do estímulo elétrico por meio da rede especializada His-Purkinje é altamente eficiente para o coração, preservando o acoplamento normal entre a condução elétrica e a contração ventricular. Zanon et al. ${ }^{28}$ ressaltam que a ativação ventricular obtida pela PHP pode ser aceita como resultante do recrutamento do feixe de $\mathrm{His}^{30} \mathrm{e}$ demonstram que a PHP pode ativar fisiologicamente o VE, de forma semelhante à intrínseca, se conduzida por meio de um sistema His-Purkinje íntegro. Os autores avaliaram a contração do VE através da estimulação de zonas distintas do septo IV e registraram que, durante a ECA da região parahissiana (PHP), a sequência de ativação e contração resultante foi similar à nativa. A normalização (ou quase normalização) da condução intraventricular com PHP pode ser explicada através de alguns conceitos anatômicos e fisiológicos do sistema de condução e das particularidades dos seus ramos direito e esquerdo. Ao contrário do ramo direito, que não estimula o septo IV até o momento que atinge o músculo papilar anterior do VD, o ramo esquerdo tem conexões precoces com o septo IV que permitem a transmissão dos impulsos do ramo esquerdo para o septo e vice-versa. Além de explicar o porquê de a ativação septal começar no lado esquerdo, isso também explica sua expressão eletrocardiográfica (ondas Q em DI e V6 e R em V1). Quando a PHP é realizada, o estímulo artificial, de maior intensidade e maior input elétrico (voltagem) do que o próprio impulso fisiológico, pode passar e avançar pelas vias normais, caso não estejam danificadas. Ainda, mas menos provável, pode "pular por cima" ou transpor um eventual bloqueio e, assim, avançar, seguindo pelo sistema eletrofisiológico e pseudonormalizando a condução. A outra possibilidade, mais provável, é que o estímulo inicialmente ative o miocárdio na cúspide do septo e, na medida em que desce pela superfície septal, se espalhe normalmente pelo sistema HisPurkinje. Isso explica o alargamento inicial do QRS estimulado, expressando ativação inicial do miocárdio septal (simulando uma onda delta) e, em seguida, o rápido desenvolvimento do QRS, similar ao nativo, indicando a ativação do sistema específico e capturando o VE através das divisões da rede de Purkinje. ${ }^{31}$

A correlação entre ECA PHP, ativação homogênea, sincronia cardíaca e análise da variância espacial do QRS pelo limeS também foi demonstrada por Bonomini et al. ${ }^{22,32}$ Segundo os autores (desenvolvedores do método Synchromax ${ }^{\circledR}$, EXO S.A., Buenos Aires, Argentina), através de análise conjugada 
de variáveis como direção do impulso elétrico (da base para o ápice ou vice-versa), duração do QRS, volume e simetria ponto a ponto das curvas obtidas entre as derivações DII e V6, é possível determinar, por meio de processamento matemático instantâneo, o tempo máximo de ativação e o registro de atrasos na propagação elétrica parietal de cada câmara ventricular ${ }^{22}$ (Figura 2A). Por meio da variância espacial do QRS, o limeS é elaborado com valores de 0,0 a 1,0, em que 00 é sincronia perfeita e 1,00, dissincronia completa ${ }^{12}$ (Figura 2B). A derivação DII do ECG de superfície representa a atividade do septo IV em termos do sentido e da velocidade de condução do estímulo elétrico. Da mesma forma, a derivação V6 representa a ativação da parede livre do VE. Na ausência de distúrbios de condução, a DII é positiva e apresenta duração preservada, mostrando ativação e tempo de condução fisiológicos da base do septo IV até o ápice cardíaco. Por outro lado, espera-se que a V6, ao representar o VE, seja positiva e apresente duração e volume espacial semelhantes à D2, pois segue o mesmo padrão de ativação. Graficamente, se há sincronia, observase sobreposição ideal das curvas resultantes (DII e V6 serão idênticas e homogêneas). A única explicação para esse efeito de curvas de ativação ventricular simultâneas e simétricas seria o recrutamento do sistema de condução intrínseco e contração ventricular coordenada e homogênea (sincronia).

Um estudo randomizado, cruzado e duplo-cego ${ }^{33}$ mostrou que a PHP preserva a FEVE e a sincronia mecânica quando comparada à estimulação septal miocárdica do VD em pacientes com bloqueio atrioventricular de alto grau, QRS estreito e FEVE $<0,40$. Kronborg et al. concluem que, nesses pacientes selecionados, não é esperado remodelamento ventricular significativo ou IC decorrentes da PHP. ${ }^{33}$ No mesmo contexto, outro estudo randomizado comparou 6 meses de PHP com 6 meses de estimulação apical do VD em 16 pacientes com fibrilação atrial crônica e ablação do nó AV. Os pacientes submetidos a PHP apresentaram redução da dissincronia IV, melhora na classe funcional, aumento significativo de rendimento no teste de caminhada de 6 minutos e diminuição das regurgitações mitral e tricúspide. ${ }^{34}$

\section{Técnica parahissiana PHP e NS-HBP: resultados similares de sincronia}

A natureza desenhou o sistema de condução cardíaco para ativar os ventrículos do endocárdio para o epicárdio, da base para o ápice e da direita para a esquerda, considerado o "eixo fisiológico" ${ }^{13}$ A presença de contração simultânea, homogênea, coordenada e simétrica demonstra sincronia. Nossos resultados confirmam que a captura comprovada do feixe de His (grupo DDD-His) resulta em contração ventricular sincrônica, idêntica à obtida durante o ritmo intrínseco, reforçando o papel da ECA do feixe de His como o padrão-ouro. A análise da variância espacial do QRS ratificou, da mesma forma, a presença de sincronia ventricular pela demonstração de limeS $<0,4$ para todos os pacientes do grupo DDD-His, exceto um (limeS $>0,7$ ). Nesse paciente, houve um microdeslocamento do eletrodo da posição do His, que passou a capturar o miocárdio septal adjacente uma complicação possível dessa técnica. ${ }^{10}$ Quando há ativação miocárdica, similarmente ao que ocorre na ECA "convencional" do VD, tanto apical quanto puramente muscular septal, a condução elétrica se faz através do tecido inespecífico (captura miocárdica), fora da rede especializada de His-Purkinje. ${ }^{2,28}$ Essa modalidade indesejada de ECA apresenta um padrão ECG característico, ${ }^{26}$ e o resultado mecânico é uma perda da eficácia da contratilidade ventricular. Conforme exposto anteriormente, a magnitude da dissincronia é demonstrada analiticamente pelo limeS na faixa próxima do valor 1.

O ponto primordial dos nossos resultados está na demonstração de que todos os pacientes do grupo DDD-Var que partiram de parâmetros de dissincronia (limeS >0,7 a 1), após o estabelecimento de PHP, recuperaram a ativação (sincronia) homogênea dos ventrículos (92,1\% com limeS < 0,4; o restante, limeS $=0,4$ a 0,69. Variação mediana do limeS no grupo DDDVar = -74). Todavia, reforçando ainda mais as semelhanças entre a ECA PHP e a NS-HBP, o sentido da ativação elétrica ventricular produzida na totalidade dos casos para ambos os grupos se enquadrou nas categorias de eixo fisiológico, na verificação integral dos critérios de Mala et al. ${ }^{25}$ e eixo provavelmente fisiológico, na falta de um dos critérios.

\section{Duração dos QRS estimulados}

O funcionamento adequado da "bomba cardíaca" depende de um sistema eletromecânico bastante coordenado (síncrono). Anormalidades de condução, como a provocada pela estimulação puramente miocárdica ventricular, causam dissincronia, que pode ter consequências deletérias (miocardiopatia associada à ECA). ${ }^{3,4,35}$ A PHP utilizada neste estudo, apesar de ter corrigido a dissincronia em praticamente todos os pacientes, o fez à custa de um alargamento significativo do QRS (Figura 8). Mesmo assim, nenhum caso superou o valor crítico de $150 \mathrm{~ms} .{ }^{19,28} \mathrm{Um}$ estudo conduzido por Zhang et al. ${ }^{9}$ que objetivou avaliar o efeito agudo da ECA seletiva do feixe de His (S-HBP), da NS-HBP e da estimulação muscular do septo IV direito na sincronia elétrica e mecânica do VE, mostrou que tanto a S-HBP quanto a NS-HBP poderiam restaurar a contração elétrica fisiológica e a sincronia mecânica ventricular. Ratifica-se que a estimulação do feixe de His, em qualquer das suas modalidades, pode manter a ativação ventricular nativa através do sistema de condução intrínseca, o que é comprovadamente mais fisiológico e caracterizado por melhores indicadores de sincronia cardíaca do que pacientes com ECA puramente muscular. ${ }^{9}$ Contudo, em vários estudos, a duração do QRS no ECG de 12 derivações tem sido usada como um marcador indireto de sincronia elétrica, e a prolongação do QRS, intrínseco ou estimulado, foi associada a risco aumentado de insuficiência cardíaca. ${ }^{8,36}$ Em nosso estudo, houve diferença a favor do DDD-His quando comparado ao DDD-Var (PHP) em relação à menor duração do QRS estimulado; porém, em ambas as técnicas, percebeu-se alargamento significativo e na mesma magnitude quando comparado ao QRS intrínseco. Esse resultado também esteve presente no estudo de Zhang et al. ${ }^{9}$ durante a ECA de baixa voltagem com NS-HBP, em que, apesar de ter demonstrado melhor sincronia elétrica e mecânica do que a estimulação septal muscular, o QRS estimulado era mais largo do que o QRS intrínseco.

Todavia, nossos achados destacam que a magnitude da variação do QRS pré e pós-procedimento entre os grupos não foi significativa $(p=0,436)$, ressaltando que a NS-HBP (grupo DDD-His) comprovadamente capturava o sistema elétrico nativo. A explicação seria que o estímulo próximo ao feixe de His e não diretamente sobre ele (ECA fisiológica indireta) produz 
complexos QRS fusionados. Observou-se alargamento inicial (onda pseudodelta) atribuível à captura concomitante do tecido muscular perihissiano, resultando em duas frentes despolarizantes que se fusionam. Uma frente recruta o sistema intrínseco e ativa o VE pelo ramo esquerdo nativo, e a outra trafega brevemente pelo miocárdio do septo IV adjacente ao His até encontrar e ativar o sistema de condução pelo lado direito, similarmente ao observado nas síndromes de pré-excitação com vias acessórias parahissianas. ${ }^{31}$ Contudo, embora a presença de dissincronia mecânica seja frequente em pacientes com complexos QRS largos, a largura do QRS por si só não parece ser um marcador eficiente de diagnóstico de dissincronia. ${ }^{12,13,22} \mathrm{O}$ eixo de ativação e a dispersão morfológica da despolarização ventricular seriam características mais marcantes, como mostrado por Bonomini et al. ${ }^{22}$ Essa importante mudança de paradigma foi confirmada em uma publicação comparando o método de variância espacial do QRS com a ecocardiografia. O ECG com análise de variância teria sensibilidade superior e relevante valor preditivo negativo para a detecção de dissincronia mecânica quando comparado somente à duração do QRS do ECG convencional ${ }^{32}$ sugerindo que importa mais a "coordenação" do que a duração do QRS.

\section{Estimulação cardíaca fisiológica com ambas as técnicas?}

Publicações recentes ${ }^{26,27,37}$ permitem discernir, no ECG, as diferenças entre ativação puramente miocárdica e a captura direta ou indireta do sistema de condução intrínseco (Figura 3). A análise desses critérios, como neste estudo, reduziria o risco de incorretamente identificar a captura do miocárdio inespecífico como PHP, o que, em alguns casos, está associada a resultados clínicos semelhantes à ECA convencional do VD. ${ }^{26,28}$ Quando a condução elétrica trafega pelo tecido muscular não especializado, ativa o septo IV de forma anômala e leva ao atraso acentuado da ativação da parede lateral do VE, ocasionando alteração morfométrica e deformação do QRS. Pela aplicação exaustiva desses princípios eletrocardiográficos, ${ }^{26,27}$ não houve diferença significativa $(p=0,999)$ entre os grupos estudados. De interesse, no grupo DDD-Var, a ausência de entalhe na derivação V1 e o tempo entre o estímulo (espícula) até o RWPT em V6 <100 ms foram as duas características mais marcantes ao conceito de ECA fisiológica, assemelhando a ECA PHP à NS-HBP. Em contrapartida, no DDD-His, talvez corroborando a manifestação de NS-HBP, o achado que mais afastou o diagnóstico de ECA fisiológica foi RWPT > 100 ms. Contudo, isso é explicado pelo tempo de propagação do estímulo até a penetração e captura do feixe de His. O método de análise da variância espacial do QRS (Synchromax ${ }^{\circledR}$ ) corrobora de forma coerente essa sincronia, comparável ao NS-HBP e determinando um limeS <0,4 com sobreposição das curvas de ativação.

\section{Segurança e eficácia da PHP}

Devido às características da curva de aprendizado relacionada à técnica da ECA do feixe de His, preferiu-se aplicar essa estratégia em pacientes com DNS e condução atrioventricular preservada. Os pacientes com bloqueios intra e infra-His apresentam desafios adicionais à essa técnica e, muitas vezes, precisam que a estimulação do VD seja garantida por um segundo eletrodo de backup (maior consumo de recursos e maior risco de complicações).
O posicionamento do eletrodo VD nas regiões proximais do septo IV na busca de ativação parahissiana é mais simples e de fácil reprodutibilidade, exequível em qualquer serviço que realize implantes de MPd com o auxílio da anatomia radiológica. ${ }^{19}$ Nossos resultados confirmam que a PHP é viável e especialmente segura para pacientes dependentes da ECA, grupo no qual a estimulação por captura do His pode ser mais desafiadora. ${ }^{10}$ Ainda, a PHP possui o benefício de apresentar uma curva de aprendizado mais breve e um tempo de exposição à Rx durante o procedimento significativamente menor (Figura 9).

Observa-se, também, que a programação do MPd e a solução de problemas intraoperatórios relacionados à ECA direta do His (HBP) podem ser um obstáculo. ${ }^{37}$ Em contrapartida, para o grupo de pacientes DDD-Var, foram registrados parâmetros eletrônicos favoráveis, como ondas R de amplitude significativamente melhor (Figura 10). É possível vislumbrar que a PHP, realizada com eficácia e segurança, supera algumas clássicas inconveniências da ECA fisiológica quando comparada à HBP. Hanifin et al. ${ }^{37}$ sugerem que os operadores que praticam HBP necessitam de um treinamento específico para a resolução de adversidades tanto durante o procedimento quanto durante os ajustes de programação do dispositivo. Naturalmente, esse cenário aumenta o consumo de recursos de saúde. ${ }^{37}$

Durante a HBP, a baixa amplitude da onda R pode ser uma realidade indesejada e, dessa forma, predispor a problemas de detecção da atividade elétrica intrínseca, acarretando disfunção do MPd e conflitos de programação. ${ }^{37-39}$ Observa-se, também, que a captura direta do feixe de His habitualmente requer maior energia de saída (voltagem) e, consequentemente, resulta em drenagem precoce da bateria do gerador. ${ }^{10} \mathrm{Em}$ nosso estudo, foi feita procura exaustiva por parâmetros adequados de ECA, o que pode ter interferido no aumento do tempo de Rx nesse grupo.

\section{Revisão da classificação da estimulação cardíaca fisiológica direta e indireta}

Nosso estudo é pioneiro em comparar a sincronia eletromecânica do VE através do processamento instantâneo da variância espacial do QRS entre pacientes submetidos a NS-HBP e aqueles submetidos a ECA PHP. Considerando que a ausência de diferença não significa estritamente a equivalência, propomos, a partir dos nossos achados, reclassificar a ECA fisiológica com base no grau de envolvimento (captura) direto ou indireto do sistema His-Purkinje. Dessa forma, a ECA fisiológica direta integraria a captura rigorosa sob mapeamento do sistema elétrico intrínseco, com demonstração do recrutamento do feixe de His (S-HBP - His direto seletivo) ou um de seus ramos (estimulação do ramo esquerdo - técnica deep-septal). A ECA fisiológica indireta seria representada pela NS-HBP e pela PHP, sob comprovação de ativação ventricular rápida e homogênea (sincrônica), apresentando, no entanto, captura breve, parcial e variável do miocárdio perihissiano (configurando onda pseudodelta no ECG). Apropriadamente, a ECA fisiológica indireta contemplaria a variante anatômica intrasseptal do His (tipo II), 24,37,40,41 que produz NS-HBP em quase todos os casos e pode estar presente em mais de 30\% das apresentações anatômicas.

Por fim, a estimulação direta do feixe de His é o padrãoouro para preservar o padrão de ativação fisiológica. As formas indiretas não seletivas e a PHP são variantes que, analogamente, 
como comprovado neste estudo, preservam a sincronia ventricular contrátil, evitando os potenciais efeitos deletérios da ECA convencional. ${ }^{9,12}$

Conforme apresentado, a ECA fisiológica indireta do tipo PHP é capaz de recrutar precocemente o sistema de condução intrínseco. ${ }^{31}$ Estamos diante de uma modalidade de ECA interessante e promissora, e o uso de ferramentas como a análise de sincronia pela variância espacial do QRS (limeS, Synchromax $®$ ) torna o método mais facilmente reprodutível e eficaz.

\section{Limitações}

Este estudo contemplou uma série relativamente pequena de pacientes com indicações heterogêneas de ECA, muito embora os testes para a análise de sincronia tenham sido realizados com sobre-estimulação e captura ventricular uniforme (modo $\mathrm{VII}$ ). Além disso, é um estudo unicêntrico com limitações na análise retrospectiva dos dados. Ainda, deve-se considerar que, apesar das específicas condições metodológicas, a conferência de sincronia foi por método indireto, não tendo sido consideradas outras variáveis que possam alterar a condução elétrica cardíaca. Por fim, o efeito real da manutenção da sincronia, evitando a miocardiopatia pela ECA, só poderia ser avaliado através de seguimento de longo prazo, o que não era o objetivo deste estudo. Seu foco e fortaleza estão na comparação de estratégias de ECA fisiológica durante o processo perioperatório de implante.

\section{Conclusão}

Este trabalhoevidencia a manutenção da sincronia cardíaca com a PHP similar à NS-HBP, agrupando-as em uma nova classificação: ECA fisiológica indireta. Embora essa estratégia seja promissora e atraente, apresentando-se como uma alternativa válida e comparável quando realizada com rigor metodológico, tanto o emprego da análise eletrocardiográfica da variância

\section{Referências}

1. Cho SW, Gwag HB, Hwang JK, Chun KJ, Park KM, On YK, et al. Clinical Features, Predictors, and Long-Term Prognosis of Pacing-Induced Cardiomyopathy. Eur J Heart Fail. 2019;21(5):643-51. doi: 10.1002/ ejhf.1427.

2. Safak E, Ince H, Gkouvatsou L, Schultheiss HP, Ortak J, Caglayan E, et al. Pacing-Induced Cardiomyopathy in Chronic right Ventricular Apical Pacing: AMidterm Follow-Up Study. Eur J Med Res. 2019 Jul;24(1):23. doi: 10.1186/ s40001-019-0386-5.

3. Matusik PT. Adverse Clinical Outcomes Related to Right Ventricular Pacing. Eur Heart J. 2019;40(20):1586-88. doi: 10.1093/eurheartj/ehz279.

4. Ferrari AD, Borges AP, Albuquerque LC, Sussenbach CP, Rosa PR, Piantá RM, et al. Cardiomyopathy Induced by Artificial Cardiac Pacing: Myth or Reality Sustained by Evidence? Rev Bras Cir Cardiovasc. 2014;29(3):402-13. doi: 10.5935/1678-9741.20140104.

5. Ferrari ADL. Avaliação de Dessincronia Atrioventricular em Portadores de Marca-Passo Bicameral Devido a Doença do Nó Sinusal e Bloqueio Atrioventricular de Primeiro Grau [dissertation]. São Paulo: Universidade de São Paulo; 2017. doi: 10.11606/T.98.2017.tde-05102017-074636.

6. Zanon F, Pastore G, Marcantoni L. His Bundle Pacing: The Myth is Approaching Standard Medical Care. Rev Esp Cardiol. 2020;73(8):611-14. doi: 10.1016/j.rec.2020.01.010. espacial do QRS quanto a comprovação indireta de captura do sistema de condução, como neste estudo, precisam ser validados, como qualquer nova tecnologia ou procedimento, com novos estudos e um número maior de pacientes.

\section{Contribuição dos autores}

Concepção e desenho da pesquisa: Di Leoni Ferrari A; Obtenção de dados: Di Leoni Ferrari A, Gazzoni GF, LM Domingues, Willes JCF, Cabral GC, Ferreira FVC, Lodi LO; Análise e interpretação dos dados: Di Leoni Ferrari A, LM Domingues; Redação do manuscrito: Di Leoni Ferrari A, LM Domingues, Reis G; Revisão crítica do manuscrito quanto ao conteúdo intelectual importante: Di Leoni Ferrari A, Reis G.

\section{Potencial conflito de interesse}

Não há conflito com o presente artigo

\section{Fontes de financiamento}

O presente estudo não teve fontes de financiamento externas.

\section{Vinculação acadêmica}

Não há vinculação deste estudo a programas de pósgraduação.

\section{Aprovação ética e consentimento informado}

Este estudo foi aprovado pelo Comitê de Ética da Pontifícia Universidade Católica do Rio Grande do Sul sob o número de protocolo 11/05664. Todos os procedimentos envolvidos nesse estudo estão de acordo com a Declaração de Helsinki de 1975 , atualizada em 2013. O consentimento informado foi obtido de todos os participantes incluídos no estudo.

7. Cantù F, De Filippo P, Cardano P, De Luca A, Gavazzi A. Validation of Criteria for Selective his Bundle and Para-Hisian Permanent Pacing. Pacing Clin Electrophysiol. 2006;29(12):1326-33. doi: 10.1111/j.15408159.2006.00543x.

8. Patel B, Garg J, Chaudhary R, Sablani N, Gupta R, Shah M, et al. Hi Bundle Pacing: Hemodynamics and Clinical Outcomes. Cardiol Rev. 2018;26(4):201-6. doi: 10.1097/CRD.0000000000000191.

9. Zhang J, Guo J, Hou X, Wang Y, Qian Z, Li K, et al. Comparison of the Effects of Selective and Non-Selective His Bundle Pacing on Cardiac Electrical and Mechanical Synchrony. Europace. 2018;20(6):1010-17. doi: 10.1093/ europace/eux120.

10. Keene D, Arnold AD, Jastrzebski M, Burri H, Zweibel S, Crespo E, et al. His Bundle Pacing, Learning Curve, Procedure Characteristics, Safety, and Feasibility: Insights From a Large International Observational Study. J Cardiovasc Electrophysiol. 2019;30(10):1984-93. doi: 10.1111/jce.14064

11. Kronborg MB, Nielsen JC. His Bundle Pacing: Techniques and Outcomes Curr Cardiol Rep. 2016;18(8):76. doi: 10.1007/s11886-016-0748-3.

12. Daniel O, Emilio L, Luis B, Analía P, Nicolás M, Mazzetti E, et al. Nove Implant Technique for Septal Pacing. A Noninvasive Approach to Nonselective His Bundle Pacing. J Electrocardiol. 2020;63:35-40. doi: 10.1016/j.jelectrocard.2020.09.008 
13. Zuloaga C. Qué Aprendimos de Sincronía Biventricular con el uso de "Syncromax" Rev Electro y Arritmias. 2015;9:38-43.

14. Kusumoto FM, Schoenfeld MH, Barrett C, Edgerton JR, Ellenbogen KA, Gold MR, et al. 2018 ACC/AHA/HRS Guideline on the Evaluation and Management of Patients With Bradycardia and Cardiac Conduction Delay: A Report of the American College of Cardiology/American Heart Association Task Force on Clinical Practice Guidelines and the Heart Rhythm Society. Circulation. 2019;140(8):e382-e482. doi: 10.1161/CIR.0000000000000628.

15. He H, Li X, Ke B, Chen Z, Han F, Zeng Y. Midrange Ejection Fraction as a Risk Factor For Deterioration of Cardiofunction After Permanent Pacemaker Implantation. J Interv Card Electrophysiol. 2019;55(2):213-24. doi: 10.1007/ s10840-019-00513-2.

16. Mateos JCP, Vargas RNA, Mateos EIP, Gimenes VM, Pachón MZC. Estimulação ventricular bifocal no tratamento da insuficiência cardíaca com miocardiopatia dilatada. Rev Bras Cir Cardiovasc 2000;15(1):44-54. doi: 10.1590/S010276382000000100007.

17. Pachón JC, Pachón El, Albornoz RN, Pachón JC, Kormann DS, Gimenes VM, et al. Ventricular Endocardial Right Bifocal Stimulation in the Treatment of Severe Dilated Cardiomyopathy Heart Failure With Wide QRS. Pacing Clin Electrophysiol. 2001;24(9 Pt 1):1369-76. doi: 10.1046/j.14609592.2001.01369.x.

18. Orlov MV, CasavantD, Koulouridis I, Maslov M, Erez A, Hicks A, etal. Permanent His-Bundle Pacing Using Stylet-Directed, Active-Fixation Leads Placed via Coronary Sinus Sheaths Compared to Conventional Lumen-Less System. Heart Rhythm. 2019;16(12):1825-31. doi: 10.1016/j.hrthm.2019.08.017.

19. Silva O Jr, Melo CS, Marra M, Correia D. Alternative Endocardial Sites For Artificial Cardiac Stimulation. Arq Bras Cardiol. 2011;96(1):76-85. doi: 10.1590/S0066-782X2011000100013.

20. Costa A, Gabriel L, Romeyer-Bouchard C, Géraldine B, Gate-Martinet A, Laurence B, et al. Focus on Right Ventricular Outflow Tract Septal Pacing. Arch Cardiovasc Dis. 2013;106(6-7):394-403. doi: 10.1016/j.acvd.2012.08.005.

21. Matsuo M, Shimeno K, Yoshiyama T, Matsumura Y, Matsumoto R, Abe Y, et al. Utility of the Combination of Simple Electrocardiographic Parameters For Identifying Mid-Septal Pacing. J Cardiovasc Electrophysiol. 2019;30(11):243340. doi: $10.1111 /$ jce. 14174 .

22. Bonomini MP, Ortega DF, Barja LD, Logarzo E, Mangani N, Paolucci A. ECG Parameters to Predict Left Ventricular Electrical Delay. J Electrocardiol. 2018;51(5):844-50. doi: 10.1016/j.jelectrocard.2018.06.011.

23. Ortega DF, Barja LD, Logarzo E, Mangani N, Paolucci A, Bonomini MP. NonSelective His Bundle Pacing With a Biphasic Waveform: Enhancing Septal Resynchronization. Europace. 2018;20(5):816-22. doi: 10.1093/europace/ eux098.

24. Sharma PS, Trohman R. An Electro-Anatomic Atlas of His Bundle Pacing: Combining Fluoroscopic Imaging and Recorded Electrograms. Card Electrophysiol Clin. 2018;10(3):483-90. doi: 10.1016/j.ccep.2018.05.009.

25. Mala A, Osmancik P, Herman D, Curila K, Stros P, Vesela J, et al. Can QRS Morphology be Used to Differentiate Between True Septal vs. Apparently Septal Lead Placement? An Analysis of ECG of Real Mid-Septal, ApparentMid-Septal, and Apical Pacing. Eur HeartJ Suppl. 2020;22(Suppl F):F14-F22. doi: 10.1093/ eurhearti/suaa094.

26. Burri H, JastrzebskiM, Vijayaraman P. Electrocardiographic Analysis for His Bundle Pacing at Implantation and Follow-Up. JACCClin Electrophysiol. 2020;6(7):883900. doi: 10.1016/j.jacep.2020.03.005.
27. Jastrzębski M, Moskal P, Curila K, Fijorek K, Kukla P, Bednarek A, et al. Electrocardiographic Characterization of Non-Selective His-Bundle Pacing: Validation of Novel Diagnostic Criteria. Europace. 2019;21(12):1857-64. doi: 10.1093/europace/euz275.

28. Zanon F, Barold SS. Direct His bundle and paraHisian cardiac pacing. Ann Noninvasive Electrocardiol. 2012;17(2):70-8. doi: 10.1111/j.1542474X.2012.00488.x.

29. Vijayaraman P. The Quest For Optimal Ventricular Pacing Site: Is The End Near? Europace. 2019 Nov;21(11):1607-08. doi: 10.1093/europace/euz203.

30. Laske TG, Skadsberg ND, Hill AJ, Klein GJ, laizzo PA. Excitation of the Intrinsic Conduction System Through His and Interventricular Septal Pacing. Pacing Clin Electrophysiol. 2006;29(4):397-405. doi: 10.1111/j.1540-8159.2006.00360.x.

31. Elizari M.Terapia de Resincronizacion Cardiaca: Cada vez mas cerca de lo ideal [Internet]. Capital Federal: Synchromax; 2020. [cited 2021 Aug 6]. Available from: https://synchromax.com/2020/09/18/webinar-terapia-resincronizacioncardiaca-cerca-de-lo-ideal/

32. Villarroel-ábrego H, Garillo R. Índice de varianza versus duración del complejo QRS para el diagnóstico de dispersión mecánica del ventrículo izquierdo. Insufic Card. 2020;15(1):2-9.

33. Kronborg MB, Mortensen PT, Poulsen SH, Gerdes JC, Jensen HK, Nielsen JC. His or Para-His Pacing Preserves Left Ventricular Function in Atrioventricular Block: A Double-Blind, Randomized, Crossover Study. Europace. 2014;16(8):1189-96. doi: 10.1093/europace/euu011.

34. Occhetta E, Bortnik M, Magnani A, Francalacci G, Piccinino C, Plebani L, et al. Prevention of Ventricular Desynchronization by Permanent Para-Hisian Pacing After Atrioventricular Node Ablation in Chronic Atrial Fibrillation. A Crossover, Blinded, Randomized Study Versus Apical Right Ventricular Pacing. J Am Coll Cardiol. 2006;47(10):1938-45. doi: 10.1016/j.jacc.2006.01.056.

35. MerchantFM. Pacing-Induced Cardiomyopathy: Just The Tip of The Iceberg? Eur HeartJ. 2019;40(44):3649-50. doi: 10.1093/eurheartj/ehz715.

36. Lustgarten DL, Calame S, Crespo EM, Calame J, Lobel R, Spector PS. Electrical Resynchronization Induced by Direct His-Bundle Pacing. Heart Rhythm. 2010;7(1):15-21. doi: 10.1016/j.hrthm.2009.09.066.

37. Hanifin JL, Ravi V, Trohman RG, Sharma PS. Permanent His Bundle Pacing: A programming and troubleshooting guide. Indian Pacing Electrophysiol J. 2020;20(3):121-128. doi: 10.1016/j.ipej.2020.04.004.

38. Israel CW, Tribunyan S. His Bundle Pacing: Troubleshooting During Follow-Up. Herzschrittmacherther Elektrophysiol. 2020;31(2):183-209. doi: 10.1007/ s00399-020-00693-9.

39. Vijayaraman P, Dandamudi G, Zanon F, Sharma PS, Tung R, Huang W, et al. Permanent His bundle pacing: Recommendations From a Multicenter His Bundle Pacing Collaborative Working Group for Standardization of Definitions, ImplantMeasurements, and Follow-Up. HeartRhythm. 2018;15(3):460-68. doi: 10.1016/j.hrthm.2017.10.039

40. Kawashima T, Sasaki H. AMacroscopic Anatomical Investigation of Atrioventricular Bundle Locational Variation Relative to the Membranous Part of the Ventricular Septum in Elderly Human Hearts. Surg Radiol Anat. 2005;27(3):206-13. doi: 10.1007/s00276-004-0302-7.

41. Mulpuru SK, Cha YM, Asirvatham SJ. Synchronous Ventricular Pacing With Direct Capture of the Atrioventricular Conduction System: Functional Anatomy, Terminology, and Challenges. Heart Rhythm. 2016;13(11):2237-46. doi: 10.1016/j.hrthm.2016.08.005 